\title{
Lattice-matched versus lattice-mismatched models to describe epitaxial monolayer graphene on $\mathrm{Ru}(0001)$
}

\author{
D. Stradi, ${ }^{1,2}$ S. Barja ${ }^{2,3}$ C. Díaz, ${ }^{1}$ M. Garnica, ${ }^{2,3}$ B. Borca, ${ }^{3}$ J. J. Hinarejos, ${ }^{3}$ D. Sánchez-Portal,${ }^{4,5}$ M. Alcamí, ${ }^{1,2}$ \\ A. Arnau, ${ }^{4,5,6}$ A. L. Vázquez de Parga, ${ }^{2,3}$ R. Miranda, ${ }^{2,3}$ and F. Martín ${ }^{1,2, *}$ \\ ${ }^{1}$ Departamento de Química, Módulo 13, Universidad Autónoma de Madrid, 28049 Madrid, Spain \\ ${ }^{2}$ Instituto Madrileño de Estudios Avanzados en Nanociencia (IMDEA-Nanociencia), Cantoblanco, 28049 Madrid, Spain \\ ${ }^{3}$ Departamento de Física de la Materia Condensada, Universidad Autónoma de Madrid, 28049 Madrid, Spain \\ ${ }^{4}$ Materials Physics Center (CSIC-UPVIEHU), Paseo Manuel de Lardizábal 5, 20018 San Sebastián, Spain \\ ${ }^{5}$ Donostia International Physics Centre (DIPC), Paseo Manuel Lardizábal 4, 20018 San Sebastián, Spain \\ ${ }^{6}$ Departamento de Física de Materiales (UPVIEHU), Facultad de Química, Apartado 1072, 20080 San Sebastián, Spain
}

(Received 12 June 2013; revised manuscript received 1 October 2013; published 2 December 2013)

\begin{abstract}
Monolayer graphene grown on $\mathrm{Ru}(0001)$ surfaces forms a superstructure with periodic modulations in its geometry and electronic structure. The large dimension and inhomogeneous features of this superstructure make its description and subsequent analysis a challenge for theoretical modeling based on density functional theory. In this work, we compare two different approaches to describe the same physical properties of this surface, focusing on the geometry and the electronic states confined at the surface. In the more complex approach, the actual moiré structure is taken into account by means of large unit cells, whereas in the simplest one, the graphene moiré is completely neglected by representing the system as a stretched graphene layer that adapts pseudomorphically to $\mathrm{Ru}(0001)$. As shown in previous work, the more complex model provides an accurate description of the existing experimental observations. More interestingly, we show that the simplified stretched models, which are computationally inexpensive, reproduce qualitatively the main features of the surface electronic structure. They also provide a simple and comprehensive picture of the observed electronic structure, thus making them particularly useful for the analysis of these and maybe other complex interfaces.
\end{abstract}

DOI: 10.1103/PhysRevB.88.245401

PACS number(s): 73.22.Pr, 68.37.Ef, 68.55.-a, 71.15.Mb

\section{INTRODUCTION}

Understanding how the electronic and structural properties of graphene are modified by its environment is of utmost importance for its potential application in future electronic devices. ${ }^{1,2}$ In particular, the interaction with metallic surfaces is a subject of ongoing research. ${ }^{3}$ Nowadays, graphene samples exhibiting a high degree of perfection can be grown in large and uniform domains by thermal decomposition of unsaturated hydrocarbons on single crystal transition metal surfaces. Indeed, the coupling with a metal surface offers the possibility to control the properties of the graphene sheet, inducing doping and other modifications in its electronic structure. ${ }^{4}$ In several cases, such as in graphene grown on $\operatorname{Ru}(0001),{ }^{5-8} \operatorname{Re}(0001),{ }^{9} \operatorname{Rh}(111),{ }^{10,11}$ and $\operatorname{Ir}(111),{ }^{12}$ the mismatch between the lattice constant of graphene and that of the substrate leads to the appearance of an additional long-range periodic superstructure, which can be rationalized in terms of a moiré pattern. ${ }^{13}$ This additional periodicity modulates both the structural and the electronic properties of the interface to an extent that depends on the strength of the interaction between the carbon monolayer and the surface. ${ }^{14}$ Theoretical calculations, performed within the framework of density functional theory (DFT), ${ }^{15}$ have played a major role supporting experiments, which have been performed aiming at shedding some light on both the electronic and geometric properties of these complex interfaces. ${ }^{16-21}$ Two radically different approaches have been followed: on the one hand, very large models (formed by more than 500 atoms), have been employed. ${ }^{16,19-23}$ In such models, the in-plane lattice constant of graphene is maintained as much as possible to its equilibrium value ( $a=2.46 \AA$ ), so that, by considering large enough unit cells, the lattice mismatch between the graphene layer and the metal permits a realistic description of the consequently formed moire pattern. These models have been particularly helpful to elucidate the basic geometrical ${ }^{16,19}$ and electronic ${ }^{17,20}$ properties of these surfaces. Nevertheless, the computational cost associated with these realistic models is huge, ${ }^{16}$ limiting somehow their applicability for more elaborate analysis. On the other hand, when the main focus has been on those properties that can be better resolved by looking at the band dispersion in the reciprocal space, which is particularly important in the case of graphene, such as the formation ${ }^{18}$ or the modification ${ }^{24,25}$ of specific electronic states, the most common approach has been that of adopting models in which a planar graphene sheet is artificially stretched to adapt pseudomorphically to the metallic substrate. The use of such simplified models has been justified in view of the fact that the graphene monolayer interacts with the metallic substrate mainly via the $\pi$ bands. In the vicinity of the Fermi level, the dispersion of these $\pi$ bands is weakly altered by moderate in-plane stretching. ${ }^{26}$ Indeed, when the mismatch between the lattice constant of the graphene and that of the substrate is very small, such as in graphene grown on $\mathrm{Ni}(111)$, a commensurate $1 \times 1$ structure is formed..$^{27,28}$ Consequently, in this case the lattice-matched models describe reliably both the electronic features ${ }^{29}$ and the geometry ${ }^{30}$ of the surface. On the other hand, when the mismatch between the two lattice constants is larger, both lattice-mismatched and lattice-matched models have been employed. ${ }^{16,17,19-21,24,25}$ However, despite the very different description that these 
two types of models make of the same system, a proper comparison assessing the limits of their applicability to resolve the same surface physical properties has not been carried out yet.

In this work, we have performed such comparison for the prototypical lattice-mismatched graphene/ruthenium interface [gr./Ru(0001)]. Its interest resides in the fact that the applicability of the lattice-matched model has been already questioned in the past. ${ }^{31,32}$ Graphene grown on $\mathrm{Ru}(0001)$ forms a well defined coincidence lattice which extends over large domains (up to $1 \mathrm{~mm}$ ), ${ }^{6-8}$ with a periodic unit cell formed by $25 \times 25$ graphene unit cells matching to $23 \times 23 \mathrm{Ru}(0001)$ unit cells, as suggested by surface $\mathrm{x}$-ray diffraction (SXRD). ${ }^{33}$ Across this large periodic cell, the interaction between graphene and $\mathrm{Ru}(0001)$ varies considerably depending on the relative position of the graphene atoms with respect to those of the underlying $\mathrm{Ru}$ substrate. Therefore, ideally, it is possible to discriminate between regions in which graphene is strongly bound to the surface, and regions in which the interaction is relatively weak. ${ }^{16,19}$ This inhomogeneous graphene/metal interaction has been found to induce a strong modulation in the geometric, as well as in the electronic properties of the surface. Experimentally, the former have been investigated using a wide range of techniques, such as scanning tunneling microscopy (STM), $5,7,8$ low-energy electron diffraction (LEED), ${ }^{34}$ surface X-ray diffraction (SXRD), ${ }^{33,35}$ and $\mathrm{He}$ atoms scattering (HAS). ${ }^{36}$ These experiments have yielded somehow contradictory results. Despite the fact that these results show similar shapes of the moiré structure, the actual range of the measured corrugations lies between $\sim 0.4 \AA$, as determined by HAS, and $\sim 1.5 \AA$, as determined by LEED. On the other hand, scanning tunneling spectroscopy (STS) has been able to resolve the localized nature of the surface electronic structure in the close proximity of, ${ }^{5,8,37}$ as well as above the Fermi level. ${ }^{18,38}$

The paper is organized as follows. Section II describes the computational methods and models employed in the present work. Section III A explains how the use of lattice-matched and lattice-mismatched models allows for an accurate analysis of the nature of the interactions determining the actual geometry of the gr./Ru(0001) surface. Section III B explains how the structural and electronic properties can be connected trough the analysis of STM topographies, and Sec. III C provides a comparison between the electronic properties of the surface obtained with the lattice-matched and the lattice-mismatched models. The main conclusions are given in Sec. IV.

\section{COMPUTATIONAL METHODS}

All the calculations have been performed using density functional theory (DFT), as implemented in the VASP code. ${ }^{39,40}$ The electronic exchange-correlation energy has been modeled using the local density approximation (LDA), as well as the generalized gradient approximation (GGA). In applying the GGA, we have used the Perdew-Burke-Ernzerhof ${ }^{41}$ (PBE) functional. The effect of $\mathrm{vdW}$ interactions has been included in the DFT/PBE calculations by means of the DFT + D2 method of Grimme ${ }^{42}$ (DFT $\left.+\mathrm{D} 2 / \mathrm{PBE}\right)$. The projector augmented wave ${ }^{43}$ (PAW) method has been used to describe the ionic cores, treating explicitly the semicore $p$ electrons of ruthenium. The single-particle Kohn-Sham Bloch states have been expanded in a plane-waves basis using a $400 \mathrm{eV}$ kinetic energy cutoff for the plane-wave expansion. The gr./Ru(0001) interface has been described either by using $1 \times 1$ models, in which the graphene has been stretched to the $\mathrm{Ru}(0001)$ lattice constant ( $a=2.73 \AA$ ), or larger models consisting of an array of $11 \times 11$ graphene unit cells matched to an array of $10 \times 10 \mathrm{Ru}(0001)$ unit cells. For the $1 \times 1$ models, the $\mathrm{Ru}(0001)$ surface has been described by employing slabs of three and five layers during total energy and electronic structure calculations, respectively. For the $11 \times 11 / 10 \times 10$ supercells, $\mathrm{Ru}(0001)$ slabs of three and five layers have also been used. For both models, the five-layer geometries have been created by adding the mirror replica of the correspondent threelayer geometry, with the mirror plane set at the bottommost $\mathrm{Ru}(0001)$ layer, in order to avoid possible spurious electronic effects arising from the presence of uncompensated dipoles, due to the use of an asymmetric slab. The geometry of the three-layer $11 \times 11 / 10 \times 10$ models has been optimized by relaxing the graphene and the topmost $\mathrm{Ru}(0001)$ layers, using a convergence criterion for the Hellmann-Feynman forces of $0.01 \mathrm{eV} / \AA^{3}$; additional relaxation of selected geometries with a criterion for the forces set to $0.005 \mathrm{eV} / \AA^{3}$ lead to variations of the optimized atomic positions below $0.015 \AA$. During the structural optimization, the interlayer distance between the two bottommost $\mathrm{Ru}(0001)$ layers has been kept fixed at the value calculated for bulk ruthenium $(d=2.077 \AA)$. The geometry obtained following this procedure agrees with that reported in the literature, obtained from more elaborate calculations in which a larger unit cell and a larger number of $\mathrm{Ru}(0001)$ layers have been used. ${ }^{22}$ To sample the $1^{\text {st }}$ Brillouin zone (BZ) of the $1 \times 1 \mathrm{gr} . / \mathrm{Ru}(0001)$ models, a Monkhorst-Pack grid $^{44}$ of $11 \times 11 \times 1 k$ points has been used for total energies (converged up to $30 \mathrm{meV} / \mathrm{C}$ atom), while a $36 \times 36 \times 1$ grid has been used to compute the density of states. For the $11 \times 11 / 10 \times 10 \mathrm{gr}$./ $\mathrm{Ru}(0001)$ supercells, only the $\Gamma$ point has been used: this ensures an equivalent sampling for the total energies obtained with the $1 \times 1$ and the $11 \times 11 / 10 \times 10$ models. A MethfesselPaxton ${ }^{45}$ smearing of $0.2 \mathrm{eV}$ has been used during the geometry optimizations and for the total energy calculations, while for the electronic structure calculations, a gaussian smearing of $0.1 \mathrm{eV}$ has been employed.

The STM topographies have been simulated by means of the Tersoff-Hamann approximation. ${ }^{46}$ The local density of states (LDOS) at the energy $\epsilon$ and position $\vec{r}$ above the surface has been calculated according to:

$$
\operatorname{LDOS}(\epsilon, \vec{r})=\sum_{m}\left|\Psi_{m}(\vec{r})\right|^{2} \delta\left(\epsilon-\epsilon_{m}\right)
$$

where $\Psi_{m}$ and $\epsilon_{m}$ are the wave function and the energy of the $m$ th Bloch state, respectively. In our case, to take into account the finite size effects due to the finite number of layers used to describe the surface, the $\delta$ function $\delta\left(\epsilon-\epsilon_{m}\right)$ has been replaced by a normal distribution: ${ }^{47}$

$$
\delta\left(\epsilon-\epsilon_{m}\right) \simeq \frac{1}{\sigma \sqrt{2 \pi}} e^{-\left(\epsilon-\epsilon_{m}\right) /\left(2 \sigma^{2}\right)}
$$

with $\sigma=0.1 \mathrm{eV}$. In the strained $1 \times 1$ models, the LDOS has been calculated over the whole unit cell considering the average of a regular grid of 30 LDOS. In the case of the 
$11 \times 11 / 10 \times 10$ model, each LDOS corresponds to an average of 30 LDOS taken in a region corresponding to a single $\mathrm{Ru}(0001)$ unit cell, centered at the different regions of the corrugated graphene moiré. In all the calculations, a vacuum gap larger than $17.5 \AA$ has been employed.

\section{RESULTS}

\section{A. Bonding properties of the gr./Ru(0001) moiré}

The morphology of $\mathrm{gr} / \mathrm{Ru}(0001)$ is determined, in first place, by the interaction between the graphene layer and the substrate at the different regions of the moiré. To explore the different regions individually, we have considered the onedimensional (1D) potential energy surface (PES) of a series of $1 \times 1$ models of this interface. The 1D-PESs have been obtained by displacing a planar graphene layer towards the $\mathrm{Ru}(0001)$ surface at regular steps of $0.1 \AA$, interpolating the points with spline functions, and extracting the position $\left(d_{\mathrm{gr} . / \mathrm{Ru}(0001)}\right)$ and depth $\left(E_{\min }\right)$ of the adsorption well from the interpolated data. By varying the position of the graphene honeycomb with respect to the high symmetry positions of $\mathrm{Ru}(0001)$, the bonding patterns encountered in the different regions of the moiré superstructure can be addressed [Fig. 1(a)]. Three different configurations have been considered: two of them are representative of the central region of the two lower areas of the graphene moiré [Figs. 1(b) and 1(c)]. In these configurations, one of the two hexagonal graphene sublattices sits above the $\mathrm{Ru}(0001)$ Top site, while the second one occupies the Hcp and the Fcc site, respectively. Conversely, the third configuration describes the higher region of the moiré [Fig. 1(d)], in which the $\mathrm{C}$ atoms sit only on Fcc and Hcp sites. In the following, these three models will be referred as $1 \times 1-($ Hcp-Top), $1 \times 1-($ Fcc-Top), and $1 \times 1-($ Fcc-Hcp), respectively.
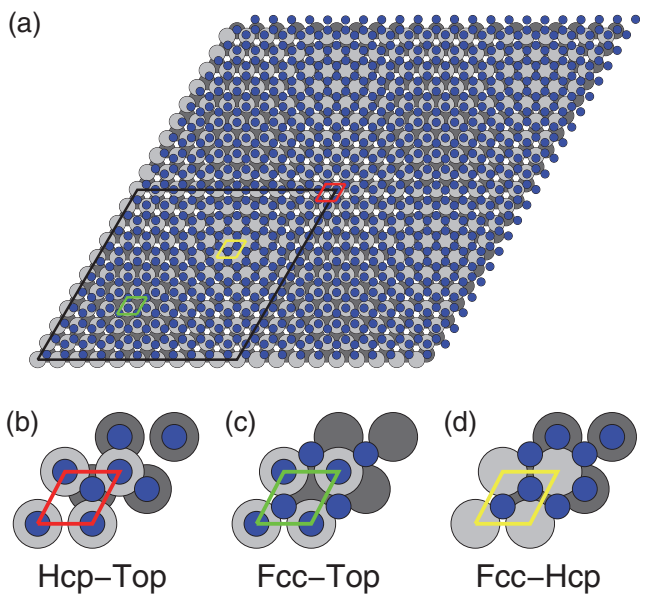

FIG. 1. (Color online) Schematic representation of (a) a $11 \times 11 / 10 \times 10$ unit cell representing the $\mathrm{gr} / \mathrm{Ru}(0001)$ moiré and (b), (c), (d) the corresponding $1 \times 1$ models: (b) $1 \times 1$-(Hcp-Top), (c) $1 \times 1$-(Fcc-Top), (d) $1 \times 1$-(Fcc-Hcp). In each panel, a total of four $2 \times 2$ unit cells is shown. Only the graphene and the topmost and topmost-1 $\mathrm{Ru}(0001)$ layers are shown. Carbon atoms, $\mathrm{Ru}(0001)$ topmost layer and topmost-1 layer atoms are printed in blue, light gray, and dark gray, respectively. The unit cells of the $1 \times 1$ models in (b), (c), (d) can be identified in (a) by the different colors of each unit cell. The unit cell of the $11 \times 11 / 10 \times 10$ model is shown in black.
Similar models have already been employed in the literature to study the interaction between graphene and $\mathrm{Ru}(0001)$. Sutter et $a l .{ }^{48}$ have shown that the LDA functional predicts the favorable adsorption of graphene for all the configurations considered (similar to those considered in this study), with only small variations in the graphene/ruthenium distance: between $d_{\text {gr. } / \mathrm{Ru}(0001)}=2.06 \AA$ for the Hcp-Top configuration and $d_{\text {gr. } / \operatorname{Ru}(0001)}=2.20 \AA$ for the Fcc-Hcp configuration. Using the PBE functional, they have found that the Fcc-Hcp configuration exhibits a repulsive behavior. On the other hand, Iannuzzi et al., ${ }^{22}$ using the PBE functional augmented with the last version of the Grimme's method, ${ }^{49}$ have found that graphene adsorbs for all the configurations, albeit the interaction is less favorable at the Fcc-Hcp one. These results suggest that, beyond the strong chemical bonding present in the low areas of the graphene moire, ${ }^{16}$ dispersion forces play a significant role in determining the structure and the energetics at the gr./Ru(0001) interface.

Figure 2 shows the $1 \mathrm{D}-\mathrm{PESs}$ obtained for the $1 \times 1$ models considered in this study, employing the LDA and the PBE functionals, in the latter case, with and without the inclusion of Grimme's correction to account for dispersion interactions. It is evident that the behavior of each 1D-PES critically depends on the adsorption site. As evidenced by the studies described in the previous paragraph, the PES obtained depends very much on the functional employed. For the $1 \times 1$-(Hcp-Top) and $1 \times 1$ (Fcc-Top) configurations [Figs. 2(a) and 2(b)], a well-defined minimum is predicted, disregarding the functional. Its position $\left[d_{\text {gr. } / \mathrm{Ru}(0001)}=2.14 \AA\right.$ for $1 \times 1$-(Hcp-Top) and $d_{\text {gr. } / \mathrm{Ru}(0001)}=$ $2.15 \AA$ for $1 \times 1-($ Fcc-Top $)]$ is very similar to the sum of the covalent radii of $\mathrm{C}$ and $\mathrm{Ru}(\sim 2.18 \AA) .{ }^{50}$ In line with this result, the well depth also suggests a covalent interaction (see

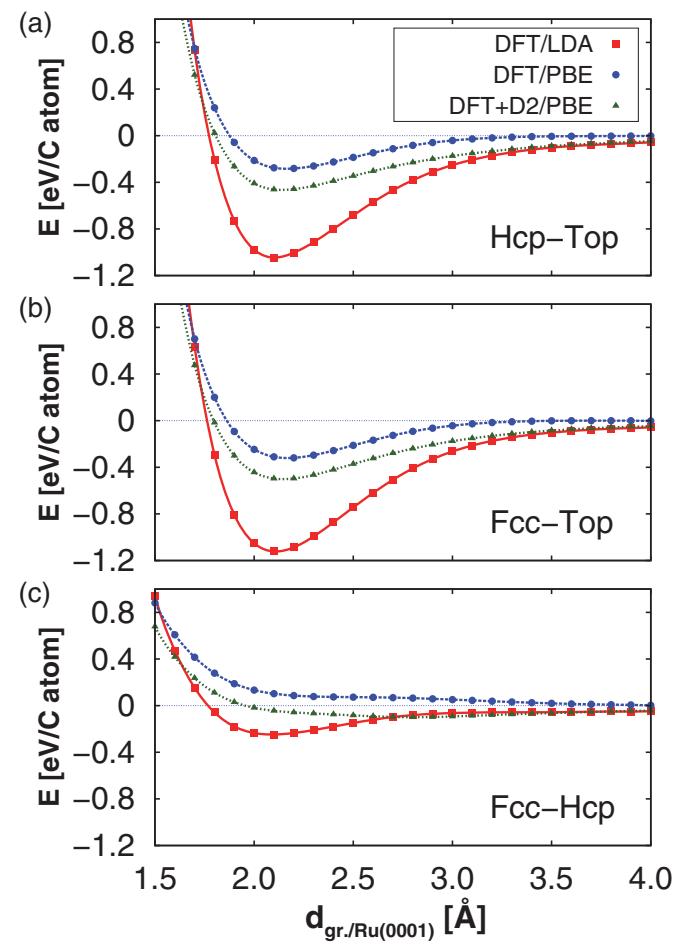

FIG. 2. (Color online) 1D-PES calculated at the DFT/LDA (red), DFT/PBE (blue), and DFT + D2/PBE (green) level of theory for (a) $1 \times 1$-(Hcp-Top), (b) $1 \times 1$-(Fcc-Top), and (c) $1 \times 1$-(Fcc-Hcp) models. 
TABLE I. Graphene/Ru(0001) distance $d_{\min }$ and adsorption energies $E_{\min }$ of the minimum energy configurations of the three $1 \times 1$ models.

\begin{tabular}{lccc}
\hline \hline & Hcp-Top & Fcc-Top & Fcc-Hcp \\
\hline & & DFT/LDA & \\
$d_{\min }(\AA)$ & 2.10 & 2.11 & 2.09 \\
$E_{\min }(\mathrm{eV} / \mathrm{C}$ atom $)$ & 1.04 & 1.12 & 0.24 \\
& & $\mathrm{DFT} / \mathrm{PBE}$ & \\
$d_{\min }(\AA)$ & 2.16 & 2.17 & - \\
$E_{\min }(\mathrm{eV} / \mathrm{C}$ atom $)$ & 0.28 & 0.32 & - \\
$d_{\min }(\AA)$ & & $\mathrm{DFT}+\mathrm{D} 2 / \mathrm{PBE}$ & \\
$E_{\min }(\mathrm{eV} / \mathrm{C}$ atom $)$ & 2.14 & 2.15 & 2.80 \\
\hline \hline
\end{tabular}

Table I). In this case, the main difference that can be observed in the 1D-PESs is that LDA predicts a much stronger binding than PBE (see Table I). In the latter case, the inclusion of $\mathrm{vdW}$ interactions results in a small increase of the adsorption wells depth, from $E_{\min } \sim 0.3 \mathrm{eV} / \mathrm{C}$ atom to $E_{\min } \sim 0.5 \mathrm{eV} / \mathrm{C}$ atom, while their position remains almost unchanged (see Table I). The situation is radically different for the Fcc-Hcp configuration [Fig. 2(c)]. In this case, the DFT/LDA 1D-PES predicts again a minimum centered at $d_{\text {gr. } / \mathrm{Ru}(0001)} \sim 2.1 \AA$, but with a considerably smaller binding energy than for the other two configurations. On the other hand, in agreement with the results of Sutter et al., ${ }^{48}$ the DFT/PBE 1D-PES is always repulsive across the whole range of graphene- $\mathrm{Ru}(0001)$ distances. Indeed, the qualitatively different shape of the two PESs computed with LDA and PBE, can be related to the description that these two functionals provide for the interlayer binding in graphite. For the latter system, LDA has been shown to provide the correct interlayer binding. ${ }^{51}$ However, this is known to arise from an unphysical tendency to bind of the exchange term, ${ }^{52}$ while vdW attraction at large distances should arise purely from correlation effects. ${ }^{53}$ This tendency is reduced in the PBE flavor of GGA, which, in fact, predicts an interlayer distance which is too large. ${ }^{51}$ In this sense, the inclusion of a semiempirical potential provides a simple, but efficient, way to correct for the absence of binding. ${ }^{51,54}$ In the present case, one can expect a similar situation, because, in $\mathrm{gr} . / \mathrm{Ru}(0001)$, graphene is an extended aromatic system adsorbed on a rather polarizable surface. Indeed, the situation changes qualitatively upon inclusion of the D2 correction: a shallow minimum $\left(E_{\min }=0.10 \mathrm{eV} / \mathrm{C}\right.$ atom $)$ appears at $d_{\text {gr. } / \mathrm{Ru}(0001)}=2.80 \AA$. Therefore, in the $1 \times 1$-(Fcc-Hcp) model, the attractive behavior of the 1D-PES is due exclusively to vdW forces. Among the methods studied here, the DFT + D2/PBE is the only one that captures properly this feature.

According to the analysis presented above, the interaction between graphene and $\mathrm{Ru}(0001)$ is very similar to that between graphene and $\mathrm{Ni}(111)$. For the latter system, two different minima have been identified at a graphene-Ni(111) separation of $\sim 2 \AA$ and $\sim 3 \AA .{ }^{29,30,55,56}$ The first one, which has been assigned to a chemisorbed state, has been found to appear only when one of the two atoms of the graphene unit cell sits above an atom belonging to the topmost layer of the metallic substrate. ${ }^{29}$ Conversely, the second minimum has been found to appear only when vdW interactions are taken into account, ${ }^{29}$ independently on the configuration of graphene over the metallic substrate. This behavior is qualitatively similar to that of the $1 \mathrm{D}-\mathrm{PESs}$ of the $\mathrm{gr} . \mathrm{Ru}(0001) 1 \times 1$ models. However, compared to graphene on $\mathrm{Ni}(111)$, in $\mathrm{gr} . / \mathrm{Ru}(0001)$ the chemisorption well calculated at the DFT + D2/PBE level of theory is $\sim 0.3 \mathrm{eV}$ deeper. ${ }^{29}$ Thus, when chemisorption occurs, the chemical interaction between graphene and the surface is stronger in the case of gr./Ru(0001) than in the case of graphene on $\mathrm{Ni}(111)$. On the other hand, the magnitude of the physisorption well, $\sim 0.1 \mathrm{eV}$, is comparable in the two systems. ${ }^{29}$ As a result, at variance with graphene on Ni(111), where the chemisorption and physisorption wells coexist in the same 1D-PES, in gr./Ru(0001) the 1D-PESs always exhibit only one of the two possible adsorption wells, depending on the atomic configuration.

Despite the significant improvement with respect to DFT/LDA and DFT/PBE in the description of the gr./Ru(0001) interface, it has to be noted that the DFT + D2 method has been developed and benchmarked exclusively for gas-phase neutral species. Therefore, its validity for the case of an aromatic extended layer deposited over a metallic surface, such as the present one, could be questionable. However, when applied to the specific case of organic molecules weakly adsorbed on metal surfaces, this method has usually been found to provide reasonable geometries, despite the fact that it tends to overestimate considerably their adsorption energies. ${ }^{57}$ This latter deficiency can be traced back to the lack of an appropriate description of the screening effects due to the delocalized environment of the metallic substrate ${ }^{58}$ To overcome this problem, rescaling the value of the metal $\mathrm{C}_{6}$ coefficients has been proposed as a pragmatic way to account for the metallic screening, thereby improving the agreement with the measured adsorption energies. ${ }^{59}$ We have therefore employed a similar approach, analyzing how varying the $\mathrm{C}_{6}$ coefficients affects the structure and energetics of the 1D-PESs. The value of the ruthenium $\mathrm{C}_{6}$ coefficients $\left[\mathrm{C}_{6}(\mathrm{Ru})\right]$ has been varied between $30.51 \mathrm{Jnm}^{6} \mathrm{~mol}^{-1}$ [value similar to the $\mathrm{Ru}-\mathrm{Ru} \mathrm{C} \mathrm{C}_{6}$ coefficient obtained by the more recent DFT-D3 method, ${ }^{49}$ $\left.\mathrm{C}_{6}(\mathrm{Ru})=31.12 \mathrm{Jnm}^{6} \mathrm{~mol}^{-1}\right]$ and $15.16 \mathrm{Jnm}^{6} \mathrm{~mol}^{-1}$. The latter value has been chosen taking into account that, for small aromatic molecules on noble metals, a value of $\mathrm{C}_{6}$ about half of the original one has resulted in geometries and adsorption energies in good agreement with the experiment. ${ }^{59}$ In addition, we have analyzed the effect of varying the $\mathrm{C}_{6}$ coefficient for carbon $\left[\mathrm{C}_{6}(\mathrm{C})\right]$, using graphite as a benchmark, because both its exfoliation energy $\left(E_{\text {graphite }}\right)$ and its interplanar distance ( $\left.d_{\text {graphite }}\right)$ have been determined experimentally $\left(E_{\text {graphite }}=\right.$ $52 \pm 5 \mathrm{meV}^{60}$ and $d_{\text {graphite }}=3.35 \AA^{61}$ ). In this case, we have varied $\mathrm{C}_{6}(\mathrm{C})$ in a more limited range, from $1.75 \mathrm{Jnm}^{6} \mathrm{~mol}^{-1}$ to $1.35 \mathrm{Jnm}^{6} \mathrm{~mol}^{-1}$, because the latter value already provides a value for $E_{\text {graphite }}$ equal to $38 \mathrm{meV} / \mathrm{C}$ atom, quite far from the experimental one. As it can be seen, reasonable agreement is obtained for the default value of $\mathrm{C}_{6}(\mathrm{C})$, $1.75 \mathrm{Jnm}^{6} \mathrm{~mol}^{-1}\left[E_{\text {graphite }}(t h)=.54.68 \mathrm{meV} / \mathrm{C}\right.$ atom and $\left.d_{\text {graphite }}(t h)=.3.25 \AA\right]$, although slightly better values are obtained using $\mathrm{C}_{6}(\mathrm{C})=1.65 \mathrm{Jnm}^{6} \mathrm{~mol}^{-1}\left[E_{\text {graphite }}\right.$ $(t h)=.50.29 \mathrm{meV} / \mathrm{C}$ atom and $\left.d_{\text {graphite }}(t h)=.3.25 \AA\right]$. Figure 3 shows the values of the well depth of the DFT + D2/PBE curves shown in Figs. 2(a)-2(c), and the 
(a)
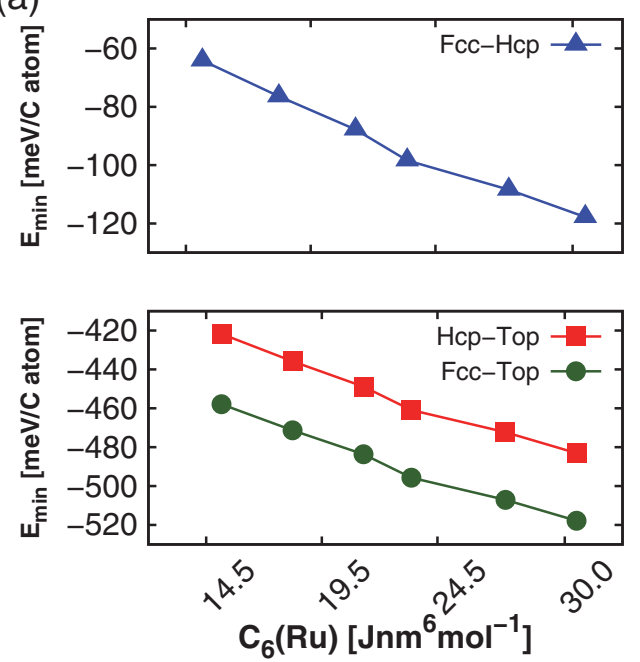

(c)
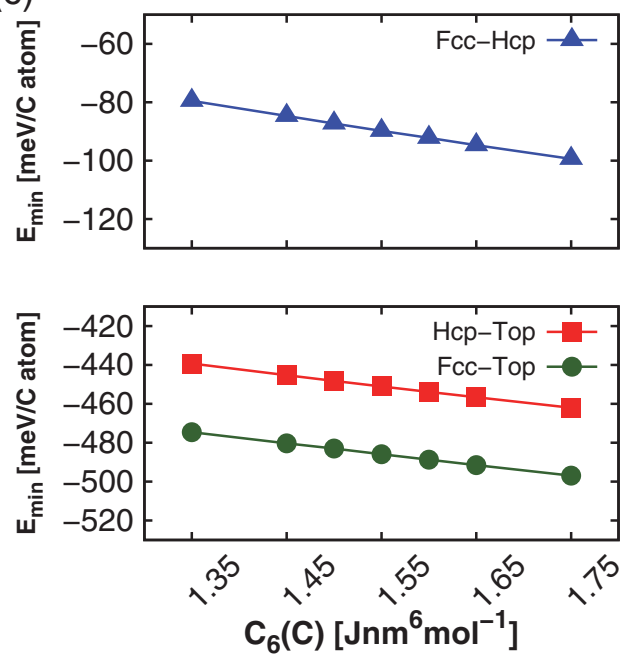

(b)
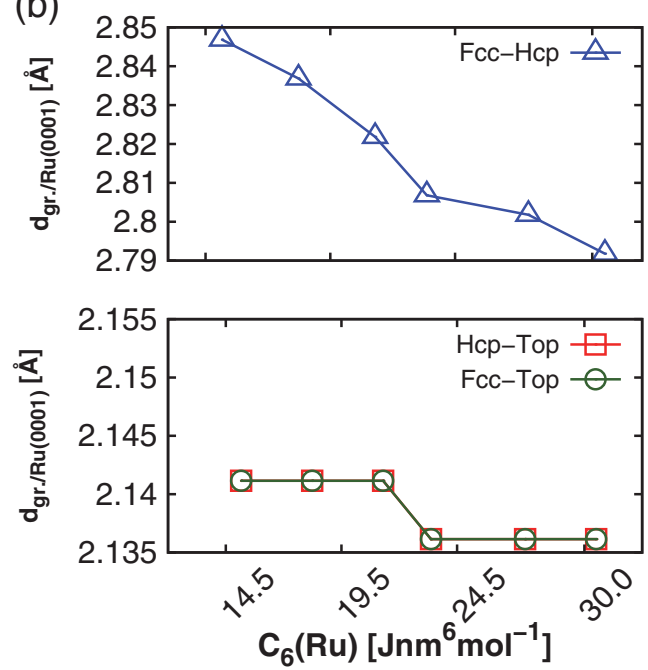

(d)
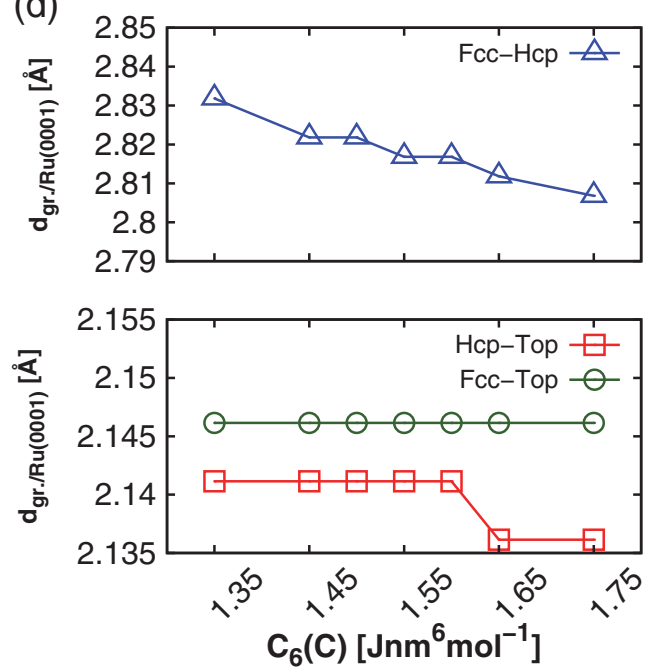

FIG. 3. (Color online) (a) Potential well depths of the 1D-PESs of the $1 \times 1$ models obtained by varying the $\mathrm{C}_{6}$ coefficient of ruthenium, with the $\mathrm{C}_{6}$ coefficient of carbon fixed at its default value. (b) Equilibrium distance between graphene and topmost Ru(0001) layer of the 1D-PESs of the $1 \times 1$ models obtained by varying the $\mathrm{C}_{6}$ coefficient of ruthenium, with the $\mathrm{C}_{6}$ coefficient of carbon fixed at its default value. (c), (d) Same as (a), (b) but varying the $\mathrm{C}_{6}$ coefficient of carbon, while maintaining the $\mathrm{C}_{6}$ coefficient of ruthenium fixed at its default value. In (a), (b), and (c), (d) the top panels show the results for the $1 \times 1-($ Fcc-Hcp) model (blue points), while the bottom panels show the results for the $1 \times 1$-(Hcp-Top) and $1 \times 1$-(Fcc-Top) models (red and green points, respectively).

values of the graphene- $\mathrm{Ru}(0001)$ distance at the adsorption well minimum, obtained by varying $\mathrm{C}_{6}(\mathrm{C})$ and $\mathrm{C}_{6}(\mathrm{Ru})$ as described above. Independently of the configuration considered, lowering each of the two $\mathrm{C}_{6}$ coefficients lead to a significant decrease of $\mathrm{E}_{\min }$. The decrease, similar for the three configurations, is more pronounced versus $\mathrm{C}_{6}(\mathrm{Ru})$ [Fig. 3(a)], than versus $\mathrm{C}_{6}(\mathrm{C})$ [Fig. 3(c)]: within the $\mathrm{C}_{6}(\mathrm{Ru})$ range (14.5-31.5) $\mathrm{Jnm}^{6} \mathrm{~mol}^{-1}, E_{\min }$ decreases by $61.3 \mathrm{meV} / \mathrm{C}$ atom, $59.9 \mathrm{meV} / \mathrm{C}$ atom and $53.7 \mathrm{meV} / \mathrm{C}$ atom for the Hcp-Top, Fcc-Top, and Fcc-Hcp configurations, respectively. These values represent a relatively small change $(\sim 15 \%)$ for the Hcp-Top and Fcc-Top configurations, but a significant change ( $\sim 50 \%)$ for the weakly bound Fcc-Hcp configuration. On the other hand, within the $\mathrm{C}_{6}(\mathrm{C})$ range $(1.35-1.75)$ $\mathrm{Jnm}^{6} \mathrm{~mol}^{-1}$, the corresponding variations are $22.7 \mathrm{meV} / \mathrm{C}$ atom, $22.3 \mathrm{meV} / \mathrm{C}$ atom, and $19.9 \mathrm{meV} / \mathrm{C}$ atom for the Hcp-Top, Fcc-Top, and Fcc-Hcp configurations, respectively. Lowering the $\mathrm{C}_{6}$ leads also to an overall increase of the graphene- $\mathrm{Ru}(0001)$ binding distance [Figs. 3(b) and 3(d)]. However, the variations of $d_{\mathrm{gr} / \mathrm{Ru}(0001)}$ are considerably smaller than those of $E_{\min }$ : within the $\mathrm{C}_{6}(\mathrm{Ru})$ range (14.5-31.5) $\mathrm{Jnm}^{6} \mathrm{~mol}^{-1}, \quad d_{\mathrm{gr} / \mathrm{Ru}(0001)}$ decreases only by $0.5 \times 10^{-2} \AA$, $0.5 \times 10^{-2} \AA$, and $5.5 \times 10^{-2} \AA$, for the Hcp-Top, Fcc-Top and Fcc-Hcp configurations, respectively. Within the $\mathrm{C}_{6}(\mathrm{Ru})$ range (1.35-1.75) $\mathrm{Jnm}^{6} \mathrm{~mol}^{-1}$, the corresponding variations are $0.5 \times 10^{-2} \AA$, $0 \AA$ (i.e., constant value) and $2.5 \times 10^{-2} \AA$ for the Hcp-Top, Fcc-Top, and Fcc-Hcp configurations, respectively. Therefore, the changes in $d_{\mathrm{gr} / \mathrm{Ru}(0001)}$ due to a modification of the $\mathrm{C}_{6}$ coefficients are always below $5 \%$ of the equilibrium binding distance. 
In summary, the results obtained with the strained $1 \times 1$ models demonstrate that the role of the vdW forces depends critically on the configuration. While dispersion forces account for about $30-40 \%$ of the binding between the graphene layer and the metal surface for the configurations corresponding to the low regions of the gr./Ru(0001) moiré, where covalent binding plays a major role, in the high regions of the moire they are the only ones responsible for the binding. In the weakly bound areas, similarly to what happens in graphite, a description based on DFT/PBE leads to a repulsive interaction, while an attractive interaction can only be recovered by introducing an additional vdW term.

X-ray diffraction and DFT calculations have shown that the electronic redistribution in the strongly bound regions leads to the in-plane expansion of the graphene honeycomb. ${ }^{34}$ Due to this effect, the weakly bound regions experience a compressive strain, which is released by outward relaxation. The delicate balance between this geometrical effect, and the attractive potential exerted by vdW interactions, determines the final corrugation of the graphene moiré. The $1 \times 1$ models do not take this effect into account, hindering the possibility to assess the role of dispersion interactions in the gr./Ru(0001) moiré structure. The $11 \times 11 / 10 \times 10 \mathrm{gr} / \mathrm{Ru}(0001)$ model [see Fig. $1(\mathrm{a})$ ] have allowed us to overcome this limitation, because in this case the different configurations are collectively taken into account within a single unit cell. As a result, the two main factors that are responsible for the actual graphene geometry, namely, the relative strength of the interaction between graphene and $\mathrm{Ru}(0001)$ with respect to the configuration, and the strain due to lateral modification of the graphene honeycomb, are considered on an equal footing. Starting from a planar graphene sheet adsorbed on the metallic surface, the geometry of this structure has been relaxed by using DFT $+\mathrm{D} 2 / \mathrm{PBE}$ (see also Ref. 19). To check the effect of the vdW interaction in the more realistic $11 \times 11 / 10 \times 10 \mathrm{gr}$. $/ \mathrm{Ru}(0001)$ model, additional calculations have been performed, in which starting from the DFT + D2/PBE geometry, the structure has been then optimized at the DFT/PBE level. Compared to models with slightly larger periodicities (e.g., $12 \times 12 / 11 \times 11^{16,22,62}$ and $13 \times 13 / 12 \times 12),{ }^{17,34}$ the $11 \times 11 / 10 \times 10$ periodicity offers the advantage that the lateral deformation needed to conform the $11 \times 11$ graphene layer to the $10 \times 10 \mathrm{Ru}(0001)$ substrate is minimum $(0.3 \%$ of its equilibrium lattice constant, compared to $1.1 \%$ and $1.8 \%),{ }^{17}$ thereby minimizing any fictitious effect due to an artificial stretching of the graphene layer. After the optimization at the DFT/PBE level, the overall corrugation of the graphene moiré has been found to be $1.60 \AA$ [Fig. 4(a)]. As discussed in Ref. 19, this value is too high to account for the apparent corrugation observed in STM experiments; however, it agrees with the value of the corrugation reported in literature for models having similar periodicities $\left(1.62 \AA,{ }^{17}\right.$ and $1.75 \AA),{ }^{62}$ which also ignored the effect of vdW interactions. The average distance between the carbon atoms in the low regions of the moiré and the topmost $\mathrm{Ru}(0001)$ layer is $2.20 \AA$, also in agreement with the distance reported in Ref. 17 (2.23 $\AA$ ) and Ref. 62 (2.3 $\AA$ ). The calculated adsorption energy (27 meV/C atom), is larger than that reported previously in the literature $\left(9 \mathrm{meV} / \mathrm{C}\right.$ atom ${ }^{17}$ and $11 \mathrm{meV} / \mathrm{C}$ atom $),{ }^{62}$ due to the explicit inclusion of the $\mathrm{Ru} p$ electrons. (a) $\mathrm{Z}_{\text {gr. }}$ (DFT/PBE)

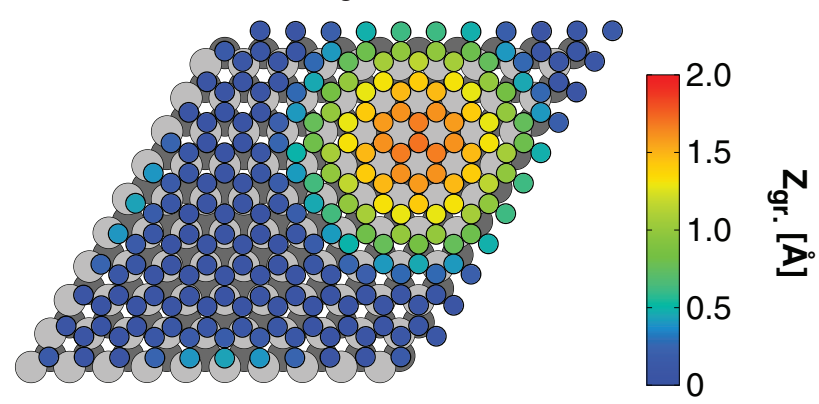

(b) $\mathrm{Z}_{\mathrm{gr} .}$ (DFT+D2/PBE)

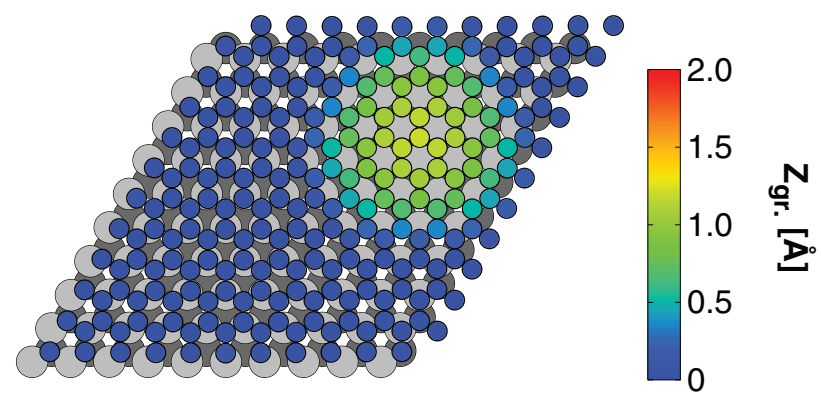

(c)

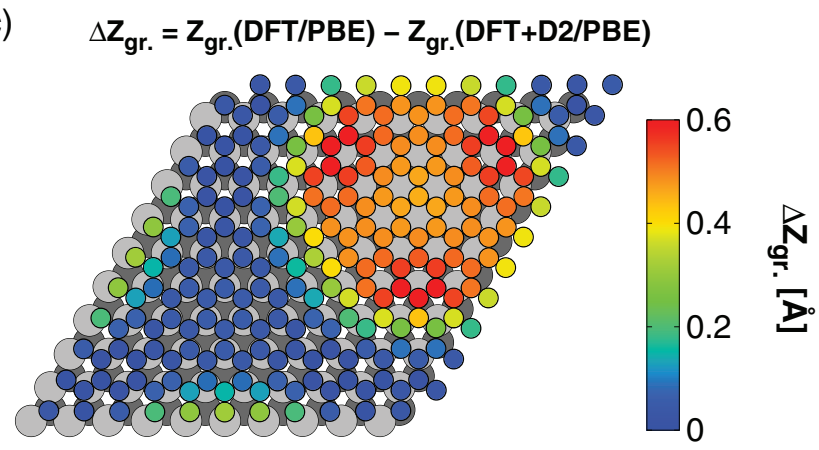

FIG. 4. (Color online) (a)-(b) Height map $\left(Z_{\text {gr. }}\right)$ of graphene atoms for the $11 \times 11 / 10 \times 10 \mathrm{gr} / \mathrm{Ru}(0001)$ supercell optimized at the DFT/PBE (a) and DFT + D2/PBE (b) level of theory. (c) Difference in vertical position $\left(\Delta Z_{\mathrm{gr}}\right)$ of the graphene atoms between the structure optimized at the DFT/PBE and DFT + D2/PBE levels of theory $\left[\Delta Z_{\mathrm{gr} .}=Z_{\mathrm{gr} .}(\mathrm{DFT} / \mathrm{PBE})-Z_{\mathrm{gr} .}(\mathrm{DFT}+\mathrm{D} 2 / \mathrm{PBE})\right]$. Ruthenium atoms of the topmost and topmost-1 layer are printed in light gray and dark gray, respectively.

In agreement with the qualitative predictions obtained with the strained $1 \times 1$ models, the inclusion of $\mathrm{vdW}$ interactions (using DFT $+\mathrm{D} 2 / \mathrm{PBE}$ ) has a significant impact on the morphology of the corrugated structure in the $11 \times 11 / 10 \times 10$ gr./Ru(0001) model. The binding energy increases about one order of magnitude, $206 \mathrm{meV} / \mathrm{C}$ atom. This variation is very similar to that obtained for the $1 \times 1$-(Hcp-Top) and $1 \times 1$-(Fcc-Top) models $(\sim 180 \mathrm{meV} / \mathrm{C}$ atom). In the low regions of the moiré, the geometry hardly varies, but in the high regions, the collective effect of $\mathrm{vdW}$ forces brings the graphene closer to the surface, reducing the graphene corrugation to only $1.19 \AA$ [Fig. 4(b)], which corresponds to a decrease of 
the ripple corrugation of about $25 \%$. Similar values for the gr./Ru(0001) moiré corrugation have recently been calculated for models with large ${ }^{22}$ and misaligned ${ }^{63}$ periodicities. This confirms that the reduction of the corrugation in lattice mismatched gr./Ru(0001) due to the vdW interaction is a robust effect, which does not depend on the particular periodicity or geometry employed. This geometrical modification can be further analyzed in terms of the difference in the vertical positions of the graphene atoms between the two optimized geometries, $\Delta Z_{\mathrm{gr} .}=Z_{\mathrm{gr} .}(\mathrm{DFT} / \mathrm{PBE})-Z_{\mathrm{gr} .}(\mathrm{DFT}+\mathrm{D} 2 / \mathrm{PBE})$ [see Fig. 4(c)]. The largest changes $\left(\Delta Z_{\text {gr. }} \sim 0.6 \AA\right)$ do not correspond to the center of the graphene ripple but to three of its six lateral sides, those pointing towards the Hcp-Top regions. This highlights the fact that the symmetry of the ripple is actually threefold, as suggested by the triangular shape of the graphene protrusions observed in the STM topographies. ${ }^{36}$

Following the inclusion of $\mathrm{vdW}$ interactions, the most pronounced changes in the geometry of the gr./Ru(0001) moiré occur in the proximity of the Fcc-Hcp region, where graphene is only weakly coupled to $\mathrm{Ru}(0001)$. Because of this, the surface electronic structure remains almost unaltered. Figure 5 shows the projected DOS (PDOS) on the $p$ orbitals of the carbon atoms at the center of the three main regions of the DFT/PBE and DFT + D2/PBE geometries of the $11 \times 11 / 10 \times 10$ model. It is evident that the overall shape of the PDOS is preserved in all the regions of the graphene moiré, although changes are slightly more pronounced in the Fcc-Hcp region, where the geometric modifications upon inclusion of $\mathrm{vdW}$ interactions are larger. Therefore, it can be concluded that $\mathrm{vdW}$ interactions have only little influence on the electronic structure of graphene/Ru(0001).

Similarly to the analysis performed with the strained $1 \times 1$ models, we have tested the influence of the $\mathrm{C}_{6}$ coefficients values on the geometry and energetics of this larger model. We have used values of $\mathrm{C}_{6}(\mathrm{C})=1.65 \mathrm{Jnm}^{6} \mathrm{~mol}^{-1}$ and $\mathrm{C}_{6}(\mathrm{Ru})=$ $15.562 \mathrm{Jnm}^{6} \mathrm{~mol}^{-1}$, in order to estimate an upper bound to the possible errors due to the use of an inaccurate value of the $\mathrm{C}_{6}$ coefficients. In this case, the corrugation rises slightly up to $1.24 \AA$, and the binding energy is reduced to $167 \mathrm{meV} / \mathrm{C}$ atom (for a more detailed analysis, see Ref. 19), showing a trend similar to that predicted by the $1 \times 1$ models. A second methodology to evaluate the possible error induced by DFT $+\mathrm{D} 2 / \mathrm{PBE}$, due to the lack of screening, is to confine the vdW interaction only to the upper layers of the surface. ${ }^{57}$ In this case, we have performed calculations in which $\mathrm{vdW}$ corrections between the graphene layer and the third topmost layer of $\mathrm{Ru}(0001)$ are switched off. Screening of the latter layer by the two upper ones should lead to the largest error due to the ABA arrangement in the three-layer $\mathrm{Ru}(0001)$ slab. The results of these modified DFT + D2/PBE calculations lead to a change of vertical displacements of less than $0.01 \AA$. Overall, the general picture provided by the default values of $\mathrm{C}_{6}$ still holds. The largest changes in the ripple corrugation that one can expect by varying the $\mathrm{C}_{6}$ coefficients within reasonable boundaries are of the order of $\pm 0.05 \AA$, a value that is much smaller than the corrugation of the ripple as well as smaller than the difference between vdW and non- $\mathrm{vdW}$ results. This analysis shows that, while the $1 \times 1$ models may not be able to capture the complex interplay between bonding and strain,
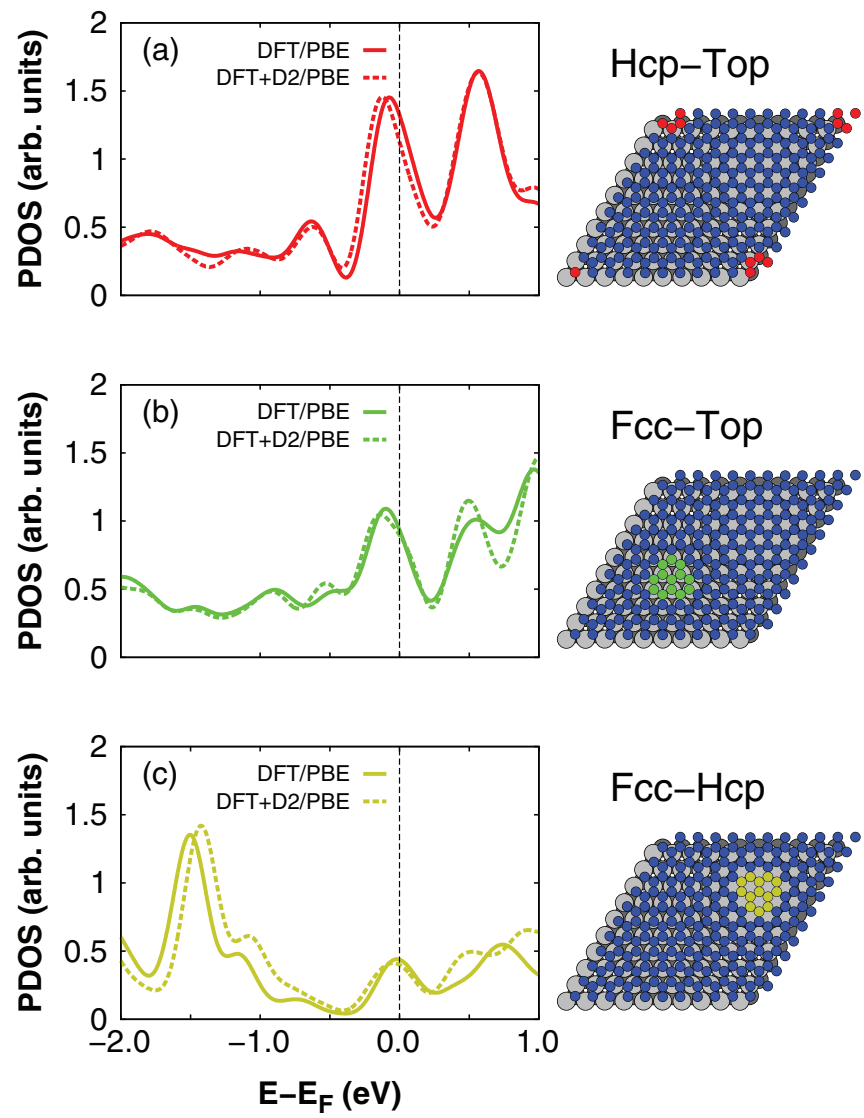

FIG. 5. (Color online) Projected density of states (PDOS) on the $p$ orbitals of the carbon atoms at the center of the Hcp-Top [(a), red], Fcc-Top [(b), green] and Fcc-Hcp [(c), yellow] regions of the $11 \times 11 / 10 \times 10 \mathrm{gr}$. $/ \mathrm{Ru}(0001)$ supercell. In (a)-(c), full lines and dashed lines correspond to the PDOS calculated using the $\mathrm{DFT} / \mathrm{PBE}$ and DFT $+\mathrm{D} 2 / \mathrm{PBE}$ geometries, respectively. The left panels in (a)-(c) highlight the atoms, which have been considered in the evaluation of each PDOS.

which determines the corrugation in $\mathrm{gr} / \mathrm{Ru}(0001)$, they are indeed useful to provide a qualitative insight of the chemical and physical interaction occurring in the different regions of the moiré.

\section{B. Effect of the structural modifications on the STM topographies}

The geometrical properties of the gr./Ru(0001) surface can be determined in terms of the apparent height measured by STM. STM, in contrast to diffraction techniques, offers the advantage that fitting is not necessary to extract the information about the surface corrugation from the rough experimental data. Thanks to the Tersoff-Hamann ${ }^{46}$ approximation, the experimental STM topographies provide a good benchmark to assess the description of the interface provided by our theoretical model, through the comparison of the experimental and the simulated apparent heights. Indeed, it has been shown that the topographical corrugation measured by STM over $\mathrm{gr} / \mathrm{Ru}(0001)$ varies depending on the bias voltage $\left(V_{\mathrm{S}}\right)$ applied between the tip and the sample. ${ }^{36,64}$ This is a clear indication that the geometrical information provided by the topographies 
is convoluted with a strong electronic effect, which prevents the determination of the precise value of the $\mathrm{gr} / \mathrm{Ru}(0001)$ corrugation only from the experimental data. Figure 6(a) shows a comparison between the measured graphene corrugation (gray dots), as a function of the bias applied between the tip and the surface, and the theoretical one, evaluated from the simulated Tersoff-Hamann topographies in the same voltage range. The simulated topographies shown in Fig. 6(a) are obtained using both the DFT/PBE geometry and a Ru(0001) slab of three layers, and the DFT + D2/PBE geometry with a $R$ (0001) slab of three or five layers. In order to mimic the typical tunneling conditions of $\sim 1 \mathrm{nA}$ and $\sim 1 \mathrm{~V}$, employed during the experiments, ${ }^{19}$ in our Tersoff-Hamann simulations we have used an electronic density contour of $1.69 \times 10^{-4} \mathrm{e}^{-} / \AA^{3}$, in the bias range $-2.0 \mathrm{eV} \leqslant V_{\mathrm{S}}<3.0 \mathrm{~V}$. At $V_{\mathrm{S}}=3.0 \mathrm{~V}$, an electron density contour ten times larger has been used, due to the finite dimensions of the vacuum employed in the periodic supercell (see discussion below). From Fig. 6, it is evident that the reduction of the corrugation of the graphene ripple due to $\mathrm{vdW}$ interactions has a direct influence on the calculated topographies. The corrugation of the DFT/PBE moiré geometry lies considerably above the measured one in the entire bias range explored $(-2.0 \mathrm{~V} \leqslant$ $V_{\mathrm{S}} \leqslant 3.0 \mathrm{~V}$ ). If the topographical images are calculated using the DFT + D2/PBE geometry, the situation improves substantially. For example, if we consider the same model, i.e., three $\mathrm{Ru}(0001)$ layers, the calculated corrugation at $V_{\mathrm{S}}=-1.0 \mathrm{~V}$ $(+1.0 \mathrm{~V})$ decreases from $1.25 \AA(0.99 \AA)$ to $0.98 \AA(0.61 \AA)$, in better agreement with the experimental one, $1.0 \pm 0.2 \AA$ $(0.5 \pm 0.2 \AA)$.

However, although the inclusion of vdW interactions improves the agreement between the simulated and the measured topographic images, the three-layer models still miss some important features present in the measured data. In particular, they are unable to capture the reduction of corrugation occurring in the close vicinity of the Fermi level between $V_{\mathrm{S}}=-0.5 \mathrm{~V}$ and $V_{\mathrm{S}}=0.5 \mathrm{~V}$, along with the contrast inversion observed in the experimental topographies above $V_{\mathrm{S}}=2.5 \mathrm{~V}$. These inaccuracies results from the discretization of the metallic continuum due to the finite number of layers employed to model the $\mathrm{Ru}(0001)$ surface. Close to the Fermi level, the number of Kohn-Sham eigenstates in this energy range is not sufficient to represent accurately the electronic density of an ideal, semi-infinite gr./Ru(0001) surface. Similarly, the discrepancies well above $E_{\mathrm{F}}$ may be due to an improper representation of the precise features of $\mathrm{Ru}(0001)$. In particular, DFT calculations performed for strained $1 \times 1$ models with $23 \mathrm{Ru}(0001)$ layers have suggested that the contrast inversion is related to the presence, in the clean $\mathrm{Ru}(0001)$ surface, of a surface resonance state located just above the projected gap at the $\Gamma$ point. ${ }^{18}$ In the low areas of the gr./Ru(0001) moire, its combination with the first image state of graphene $\left(1^{+}\right){ }^{66}$ results in a new interface state, which has been identified by STS, ${ }^{18}$ and further confirmed by two-photon photoemission (2PPE) experiments. ${ }^{67}$ While such $\mathrm{Ru}(0001)$ resonance does not appear when only three metal layers are used, it is reasonably reproduced in a five-layer slab. ${ }^{20}$ Indeed, DFT calculations employing $11 \times 11 / 10 \times 10$ $\mathrm{gr} . / \mathrm{Ru}(0001)$ models with five ruthenium layers have been able to reproduce successfully the contrast inversion ${ }^{20}$ associated (a)

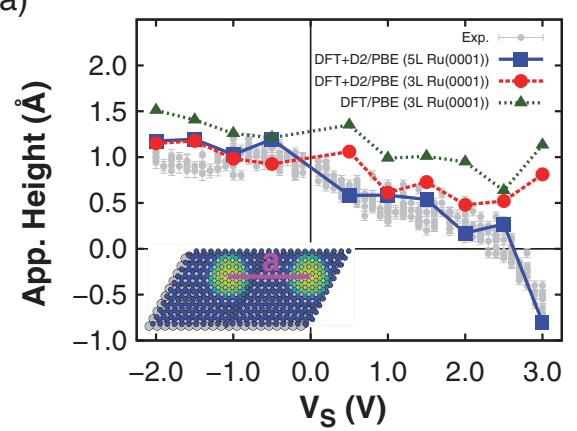

(b)

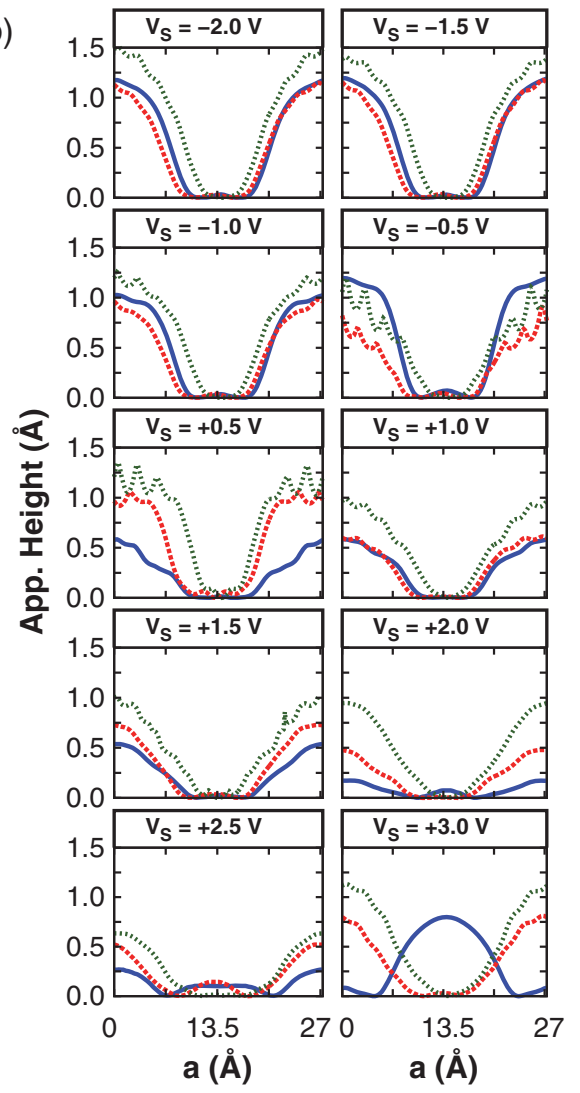

FIG. 6. (Color online) (a) Apparent height of the simulated STM topographies as a function of the bias voltage $V_{\mathrm{S}}$, for the corrugated $11 \times 11 / 10 \times 10$ model calculated at the DFT/PBE (green points) and DFT + D2/PBE level of theory with a three-layer $\mathrm{Ru}(0001)$ (red points) or a five-layer $\mathrm{Ru}(0001)$ slab (blue points). The points are connected by lines to guide the eye. The theoretical data are compared with the experimental ones (gray points). The inset indicate the moiré unit cell vector $a$ over which the corrugation has been measured both in the experimental and in the simulated topographies. (b) Line profiles of the simulated STM topographies along $a$, at different values of $V_{\mathrm{S}}$, using the DFT/PBE (green lines) geometry and $\mathrm{DFT}+\mathrm{D} 2 / \mathrm{PBE}$ geometry with a three-layer (red lines) or a five-layer $\mathrm{Ru}(0001)$ slab (blue lines).

with the presence of the new interface state. In line with these results, the entire behavior of the apparent height versus $V_{\mathrm{S}}$ is described correctly when a $\mathrm{Ru}(0001)$ slab with five layers is used [see Fig. 6(a)].

The improved description of the topographical properties becomes visible from the comparison between the profiles 
obtained from the three-layer and five-layer DFT + D2/PBE models [red and blue curves in Fig. 6(b), respectively]. At $V_{\mathrm{S}} \leqslant-1.0 \mathrm{~V}$, the profiles obtained with the two models match well, because the integration range of the DOS is sufficiently large to eliminate any spurious discretization effect. Close to the Fermi level $\left(-0.5 \mathrm{~V} \leqslant V_{\mathrm{S}} \leqslant 0.5 \mathrm{~V}\right)$, such effects become more important, since the integration range of the DOS is smaller. This is reflected in the shape of the topographic profiles, which is very irregular in the three-layer model compared to the five-layer one. At $V_{\mathrm{S}}=1.0 \mathrm{~V}$, the topographies of both models are again very similar, but at higher energies, they became clearly different. In the three-layer $\mathrm{Ru}(0001)$ slab the aforementioned surface resonance state does not appear and, therefore, the new interface state and the associated contrast inversion are absent. It should be noted that for the five-layer model, the major contribution to the surface LDOS at $V_{\mathrm{S}}=3.0 \mathrm{~V}$ comes from unoccupied electronic states that are considerably extended towards the vacuum, i.e., those associated with the new interface state. Because of this, the topographic images cannot be simulated using the same isocontour employed at lower values of $V_{\mathrm{S}}$, due to the relatively small vacuum employed in our calculations ( $\sim 20 \AA$ ). Indeed, by multiplying the isosurface value by a factor 10, meaningful topographies that can be compared with the experimental ones can be obtained. The resulting profiles show that, at this energy, the contribution from the surface topography comes mainly from a large increase in the electronic density over the low regions of the graphene moiré. As mentioned above, such feature is completely absent in the topographies obtained using the three-layer metallic slab model, which, therefore, fails to capture the corrugation inversion shown in Fig. 6(a). We have checked, by carefully examining the shape of each surface LDOS in a range of isocountours from that employed at $V_{\mathrm{S}} \geqslant 3.0 \mathrm{~V}$ to that employed at $V_{\mathrm{S}}<3.0 \mathrm{~V}$, that these conclusions are not affected by the particular choice of the isocontour values.

\section{Localized electronic states at the surface}

The fact that the five-layer $11 \times 11 / 10 \times 10 \mathrm{DFT}+\mathrm{D} 2 / \mathrm{PBE}$ geometry reproduces correctly the main topographical features observed in the experiments indicates that, in the range of energies around the Fermi level that we are considering, such model provides a good description of the electronic structure of the gr./Ru(0001) surface. In view of this, we have used it as a benchmark to verify the reliability of the strained $1 \times 1$ models, to describe those features responsible for the behavior observed in the STM topographies. For a proper comparison, slabs of five $\mathrm{Ru}(0001)$ layers have been used also for the $1 \times 1$ models. To perform our analysis, the distance between the graphene and the $\mathrm{Ru}(0001)$ topmost layer has been fixed to $d_{\mathrm{gr} . / \mathrm{Ru}(0001)}=2.2 \AA$ for the $1 \times 1$ (Hcp-Top) and $1 \times 1$-(Fcc-Top) models, and $d_{\text {gr. } / \mathrm{Ru}(0001)}=3.4$ $\AA$ for the $1 \times 1$-(Fcc-Hcp) model. These values are similar to the graphene- $\mathrm{Ru}(0001)$ distance in the low $(2.195 \AA)$ and high $(3.385 \AA)$ regions of the $11 \times 11 / 10 \times 10 \mathrm{DFT}+\mathrm{D} 2 / \mathrm{PBE}$ moire structure. Figure 7 shows the band structure of these three $1 \times 1$ models along the high symmetry directions of the two-dimensional first Brillouin zone, as well as the separate contribution of the $p$ orbitals of the two graphene carbon
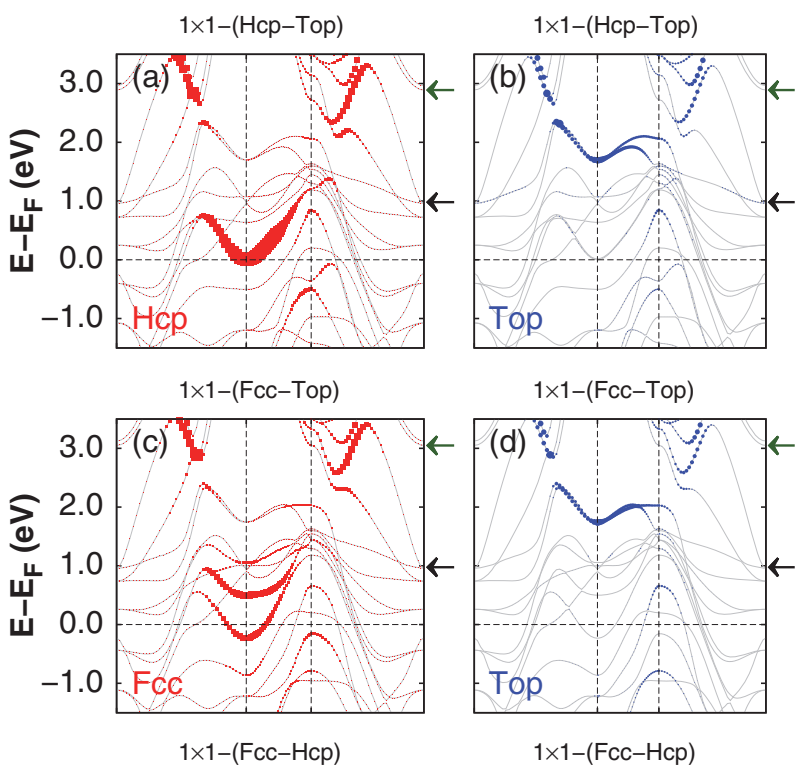

$1 \times 1-($ Fcc-Top)

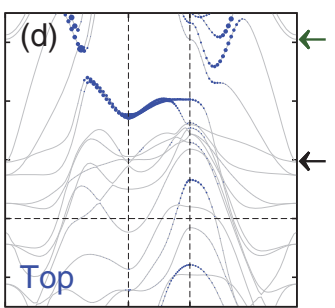

$1 \times 1-(\mathrm{Fcc}-\mathrm{Hcp})$
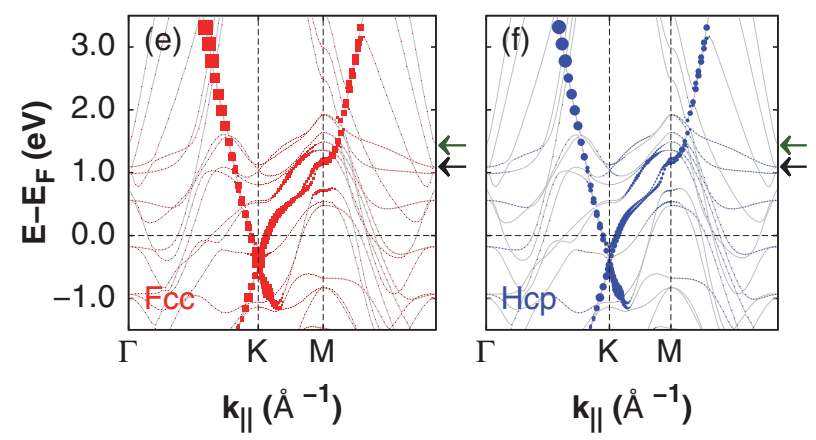

FIG. 7. (Color online) Band structure of the strained $1 \times 1$ models (see Fig. 1) along the $\Gamma \rightarrow \mathrm{K} \rightarrow \mathrm{M} \rightarrow \Gamma$ direction: (a), (b) $1 \times 1$-(HcpTop) model; (c), (d) $1 \times 1$-(Fcc-Top) model; (e), (f) $1 \times 1-($ Fcc-Hcp) model. Red squares (a), (c), (e) and blue points (b), (d), (f) indicate the weight of the graphene $p$ states of each of the two carbon atoms of graphene (the label at the bottom left of each panel indicates the adsorption site of each carbon atom). The green and black arrows at the right side of each image indicate the position in energy at the $\Gamma$ point of the band associated with the gr./Ru(0001) interface state, and with the $\mathrm{Ru}(0001)$ projected band gap at the $\Gamma$ point, respectively.

atoms to each band; it should be noted that, in the energy range considered in Fig. 7, the only relevant contribution comes from the carbon $p_{z}$ orbitals. As shown previously, ${ }_{16,24}$ the strong interaction between graphene and $\mathrm{Ru}(0001)$ in the $1 \times 1$-(Hcp-Top) and $1 \times 1$-(Fcc-Top) models leads to the disruption of the Dirac cone characteristic of freestanding graphene. Due to the different interaction of the two carbon atoms with the surface, the respective electronic bands are also modified differently [Figs. 7(a)-7(d)]. On the other hand, in the $1 \times 1$-(Fcc-Hcp) model, due to the much weaker interaction between the graphene and the metal surface, the electronic structure of the former remains almost intact, becoming weakly $n$-doped, as evidenced by the downshift below the Fermi level of the Dirac cone [Figs. 7(e)-7(f)]. Similarly, also the unoccupied electronic states above $E_{\mathrm{F}}$ exhibit a strong dependence on the $\mathrm{gr} . / \mathrm{Ru}(0001)$ distance. In the $1 \times 1$ models, the new interface state can be easily identified from the band structure, because the band associated with this state lies within 
$1 \times 1$ model

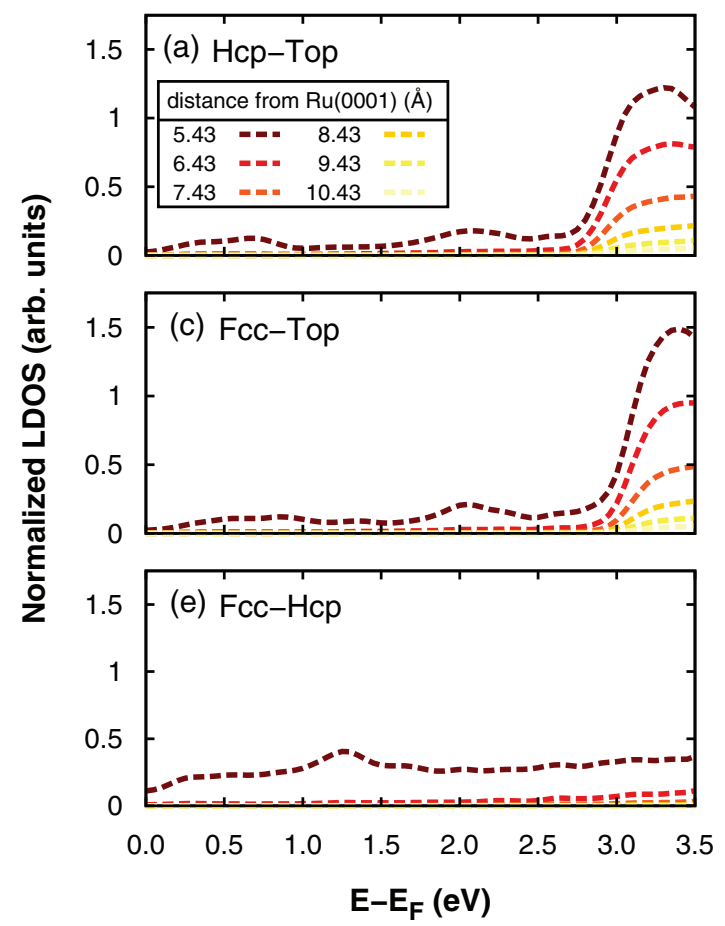

$11 \times 11 / 10 \times 10$ model
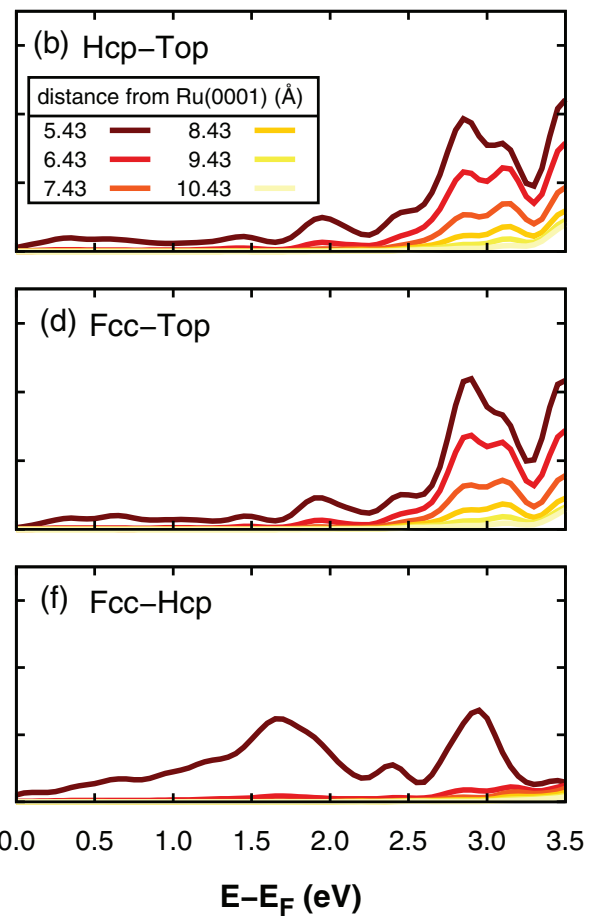

FIG. 8. (Color online) (a), (c), (e) Local density of states (LDOS) computed in the energy range $0.0 \mathrm{eV} \leqslant E-E_{\mathrm{F}} \leqslant 3.5 \mathrm{eV}$ above the $1 \times 1$-(Hcp-Top) (a), 1×1-(Fcc-Top) (c), and 1×1-(Fcc-Hcp) (e) models. (b), (d), (f) Same as (a), (c), (e), but for the Hcp-Top (b), Fcc-Top (d), and Fcc-Hcp (f) regions of the $11 \times 11 / 10 \times 10 \mathrm{gr} / \mathrm{Ru}(0001)$ supercell. The zero is set at the topmost layer of the Ru(0001) surface plane. The LDOS are printed with increasingly brighter colors depending on their distance from this plane. In each panel, the LDOSs are normalized with respect to the area of the LDOS at $5.43 \AA$.

the $\mathrm{Ru}(0001)$ projected band gap at the $\Gamma$ point. Albeit this gap is reproduced only approximatively with five $\mathrm{Ru}(0001)$ layers, its lower boundary at the $\Gamma$ point can still be identified at $E-E_{\mathrm{F}} \sim 1 \mathrm{eV}$ [black arrows in Figs. 7(a)-7(f)]. Above this energy, the first band encountered at $E-E_{\mathrm{F}}=2.89 \mathrm{eV}$ $\left(E-E_{\mathrm{F}}=3.05 \mathrm{eV}\right)$ in the $1 \times 1-($ Hcp-Top) $[1 \times 1$-(Fcc-Top)] model is associated with the interface state [green arrows in Figs. $7(\mathrm{a})-7(\mathrm{~d})]$. In the $1 \times 1-($ Fcc-Hcp) model, the larger separation between the graphene and the metal prevents the formation of the new interface state, but the $\mathrm{Ru}(0001)$ surface resonance, which is promoted to a surface state due to the presence of graphene, ${ }^{18}$ can be identified at $E-E_{\mathrm{F}}=1.43 \mathrm{eV}$ [green arrows in Figs. 7(e)-7(f)].

Performing a similar analysis in the large $11 \times 11 / 10 \times 10$ model is extremely complex, due to the downfolding of the electronic bands of both graphene and $\mathrm{Ru}(0001)$ in the first Brillouin zone. However, as already mentioned, one of the main characteristics of the new interface state is its considerable extension outside the surface. This property can be exploited to identify and compare the interface state in both models, by examining the unoccupied LDOS above the surface. Figures 8(a), 8(c), 8(e) show the LDOS corresponding to the three strained $1 \times 1$ models, calculated at increasing distances above the surface [the zero is set to the topmost $\mathrm{Ru}(0001)$ layer]. In the two models representative of the lower areas of the moiré [Figs. 8(a) and 8(c)], the most remarkable feature is an intense peak located at $E-E_{\mathrm{F}} \sim 3.25 \mathrm{eV}$ in the $1 \times 1$-(Hcp-Top) model [Fig. 8(a)] and at $E-E_{\mathrm{F}} \sim 3.4 \mathrm{eV}$ in the $1 \times 1$-(Fcc-Top) model [Fig. 8(c)]. Such peak decays very slowly into the vacuum, which indicates that the associated electronic states extend considerably outside the surface. On the other hand, the LDOS of the $1 \times 1-($ Fcc-Hcp $)$ model exhibits a completely different behavior, being essentially featureless, and decaying very rapidly when moving away from the surface. Therefore, this analysis shows that the unoccupied electronic structure above $E-E_{\mathrm{F}} \sim 3 \mathrm{eV}$ is considerably more extended towards the vacuum in those models representative of the lower regions of the graphene moiré, in agreement with the behavior that should be expected for the new interface state. Figures 8(b), 8(d), 8(f) shows the unoccupied LDOS above the surface in the three regions of the gr./ $\mathrm{Ru}(0001)$ moiré equivalent to the ones simulated with the $1 \times 1$ models [shown in Figs. 8(a), 8(c), 8(e)]. While the curve profiles are considerably more structured than those of the simple $1 \times 1$ models, most likely due to band downfolding and the moiré structure of graphene in the $11 \times 11 / 10 \times 10$ model, the overall behavior is essentially the same. In fact, also in this case a prominent peak can be identified in the Hcp-Top and Fcc-Top regions at $E-E_{\mathrm{F}} \sim 2.8 \mathrm{eV}$, in good agreement with the position of the interface state measured by 2PPE $\left(E-E_{\mathrm{F}}=2.58 \pm 0.4 \mathrm{eV}\right) .{ }^{67}$ The upshift of this feature in the $1 \times 1$ model is associated with the strain of the graphene layer, which changes the work function from $4.5 \mathrm{eV}$ to $5.2 \mathrm{eV}{ }^{48}$ Similarly to what is observed for the $1 \times 1$ models, the intensity of this peak decays very slowly into the vacuum, as it should be expected for an electronic state considerably extended outside 
(a)
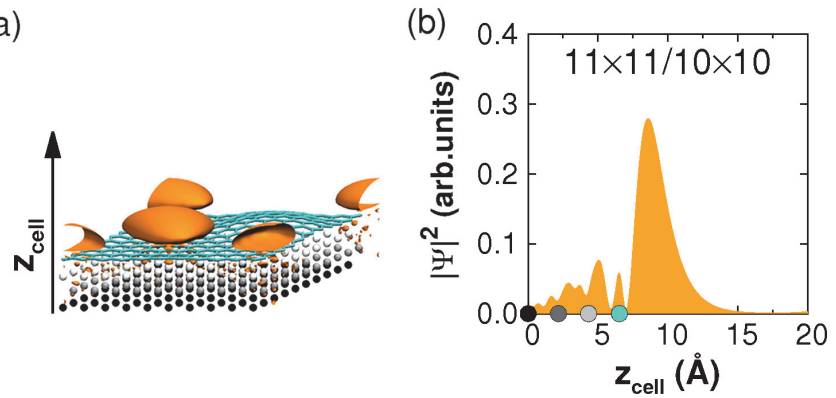

(c)
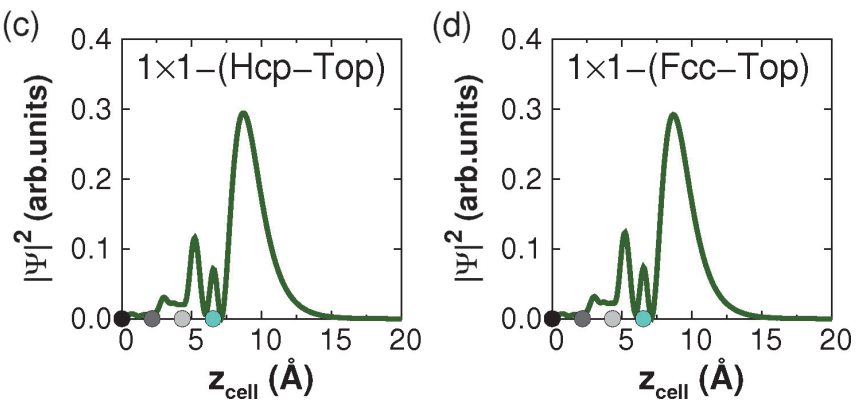

FIG. 9. (Color online) (a) Isosurface (isocontour value = $\left.1.69 \times 10^{-4} \mathrm{e}^{-} / \AA^{3}\right)$ of the lowest eigenstate associated with the gr. $/ \mathrm{Ru}(0001)$ interface state of the $11 \times 11 / 10 \times 10$ model. Only three $\mathrm{Ru}$ layers are shown for simplicity. (b) Probability density $|\Psi|^{2}$ averaged over the $x y$ plane of the eigenstate shown in (a). The graphene layer atoms are shown in cyan. The topmost, topmost-1 and topmost-2 ruthenium layers are shown in light gray, dark gray, and black. (c), (d) Probability densities $|\Psi|^{2}$ averaged in the $x y$ plane of the lowest eigenvalues associated with the $\mathrm{gr} . / \mathrm{Ru}(0001)$ interface state in the $1 \times 1$-(Hcp-Top) and $1 \times 1$-(Fcc-Top) models. In (b), (c), (d), the position of the three uppermost ruthenium layers and of the graphene layers is indicated by the dots on the $x$ axis [same colors as in (a)].

the surface. In the Fcc-Hcp region, the decay of the LDOS intensity at increasing distance from the surface is also much faster that in the low regions of the moiré. Once again, this suggests the presence of an unoccupied electronic state in the low regions of the moiré which is considerably extended towards the vacuum.

We examine now the individual eigenstates in the energy region where the peak associated with the new interface state appears $\left(2.7 \mathrm{eV} \leqslant E-E_{\mathrm{F}} \leqslant 3.3 \mathrm{eV}\right)$, in particular the one corresponding to the bottom of the interface state, which is located at $E-E_{\mathrm{F}}=2.85 \mathrm{eV}$. Its probability density $\left(|\Psi|^{2}\right)$ is shown in Figs. 9(a)-9(b). In agreement with the previous analysis, $|\Psi|^{2}$ is almost exclusively localized outside the surface over the low regions of the moiré structure [Fig. 9(a)]. By integrating $|\Psi|^{2}$ in the $x y$ plane, the individual eigenstate can be compared directly with the corresponding ones at the $\Gamma$ point of the first Brillouin zone of the $1 \times 1-($ Hcp-Top) [Fig. 9(c)] and $1 \times 1-($ Fcc-Top) [Fig. 9(d)] models, in which the three-dimensional information related to the corrugated moire is lost. The probability distributions obtained from the $1 \times 1$ models overlap almost perfectly with that calculated using the $11 \times 11 / 10 \times 10$ supercell. This confirms the robustness of the new interface state character (which does not change despite of the different models employed), as well as the accurate description provided by the $1 \times 1$ models of this feature of the gr./Ru(0001) unoccupied electronic states.

In addition to the unoccupied states just below the vacuum, the electronic structure close to the Fermi level is a second region of interest. In fact, also in this region of the electronic spectrum, the periodic variation of the interaction between graphene and $\mathrm{Ru}(0001)$ induces a strong redistribution of the surface electronic density, as revealed by the modulation of the intensity in the spatially resolved $d I / d V$ maps measured by STS. ${ }^{8}$ DFT simulations performed using the corrugated $11 \times 11 / 10 \times 10$ superstructure have shown that such modulations arise from the accumulation of electronic density below the high regions of the moiré, ${ }^{20}$ due to the doping of graphene by $\mathrm{Ru}(0001)$. Figure 10 shows the LDOSs calculated at three different heights above the graphene/Ru(0001) surface, in the region around the Fermi level $\left(-1.0 \mathrm{eV} \leqslant E-E_{\mathrm{F}} \leqslant 1.0 \mathrm{eV}\right)$ for the $11 \times 11 / 10 \times 10$ and the $1 \times 1$ models. In this case, as we are not interested in the decay of the LDOS but rather in their shape, the area under each curve has been normalized to facilitate the comparison between the LDOSs calculated at different heights. Indeed, their shape reflects the bonding characteristics of the different regions of the graphene moiré. In fact, in the low regions, Hcp-Top [Figs. 10(a)-10(c)] and Fcc-Top [Figs. 10(d)-10(f)], the LDOSs computed using the $11 \times 11 / 10 \times 10$ model and the corresponding $1 \times 1$ models agree qualitatively, reproducing the pronounced asymmetry of the STS spectra measured above and below $V_{\mathrm{S}}=0.0$ $\mathrm{V}^{8}$ This asymmetry is absent in the LDOS computed over the high regions of the $11 \times 11 / 10 \times 10$ model and in the $1 \times 1-($ Fcc-Hcp) model, in agreement with the fact that the electronic structure of graphene in these regions is only weakly perturbed. Remarkably, the best agreement between the LDOSs of the $11 \times 11 / 10 \times 10$ and the $1 \times 1$ models is obtained at the largest distance from the surface considered, $7.43 \AA$, which is comparable to the tip-surface distance in an STS experiment. At this distance from the surface, the features observed in the LDOSs are associated with the global electronic properties of the surface, rather than with those exclusively related to the graphene $\pi$ bands. ${ }^{20}$ In view of this, the close similarity between the shape of the LDOSs of the two models at $7.43 \AA$ points out that the characteristics of the measured STS spectra in gr./Ru(0001) are strongly connected with the electronic structure of the metallic substrate underneath the graphene layer, which is described almost identically in the two models.

\section{CONCLUSIONS}

In summary, we have carried out an extensive density functional theory (DFT) study on the geometry and on the electronic structure of epitaxial graphene on $\mathrm{Ru}(0001)$. We have focused on the effect of noncovalent interactions in determining the actual geometry of the graphene monolayer, as well as on the localization of electronic states induced by the presence of a moiré pattern, and compared our results with experiments available in the literature based on scanning tunnelling microscopy (STM) and scanning tunnelling spectroscopy (STS). Two different models have been used to describe the system. In the first model we have represented 


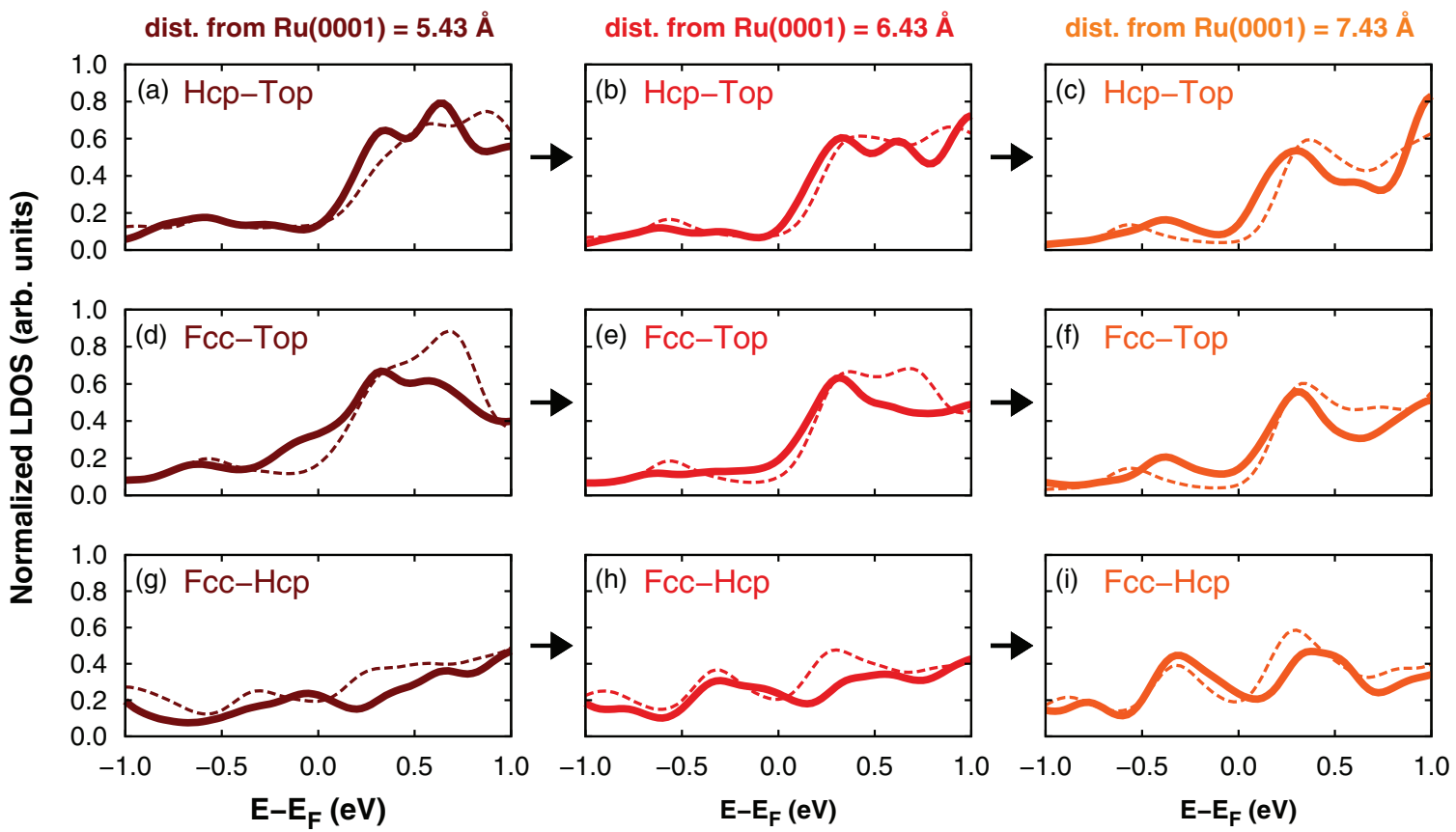

FIG. 10. (Color online) Solid lines: local density of states (LDOS) taken over the Hcp-Top (a)-(c), Fcc-Top (d)-(f), and Fcc-Hcp (g)-(j) regions of the $\mathrm{gr} / \mathrm{Ru}(0001)$ moire of the $11 \times 11 / 10 \times 10$ supercell in the energy range $-1.0 \mathrm{eV} \leqslant E-E_{\mathrm{F}} \leqslant 1.0 \mathrm{eV}$. Dashed lines: LDOS computed for the $1 \times 1-($ Hcp-Top) (a)-(c), $1 \times 1-($ Fcc-Top) (d)-(f), and $1 \times 1-($ Fcc-Hcp) $(\mathrm{g})-(\mathrm{i})$ models in the same energy range. The zero is set at the topmost layer of the $\mathrm{Ru}(0001)$ surface plane. The distance from the $\mathrm{Ru}(0001)$ topmost layer surface plane is $5.43 \AA$ (a), (d), (g), $6.43 \AA$ (b), (e), (h), and $7.43 \AA$ (c), (f), (i). Arrows indicate the direction of increasing distance from the surface. The LDOSs have been normalized to have the same area under each curve.

the gr./Ru(0001) system using a stretched graphene layer that adapts pseudomorphically to $\operatorname{Ru}(0001)(1 \times 1$ models $)$, and in the second one, an $11 \times 11$ graphene sheet adsorbed on a $10 \times 10 \mathrm{Ru}(0001)$ surface $(11 \times 11 / 10 \times 10$ model $)$, which accounts realistically for the formation of the moiré pattern, has been used. Regarding the geometries, the $1 \times 1$ models can be seen as a complementary tool to disentangle the role of the several types of interactions occurring in the moiré, and to test the accuracy of the computational model employed to describe the adsorbate surface interaction. However, they do not provide quantitative insight into the geometry of gr./Ru(0001), as they do not account for the lateral strain present in the graphene moiré. On the other hand, the two models offer essentially an equivalent description of those electronic properties that do not depend significantly on the exact geometry of the graphene monolayer. They both predict the appearance of a new interface state in the low regions of the graphene moiré, and the presence of peaks in the local density of states (LDOS) above the surface, in the proximity of the Fermi level. This may be important to address problems in which the detailed analysis of the reciprocal space is important, such as those associated with the electron dynamics at surfaces. ${ }^{47,68,69}$

\section{ACKNOWLEDGMENTS}

We thank Barcelona Supercomputing Center-Spanish Supercomputing Network (BSC-RES) and Centro de Computación Científica - Universidad Autonoma de Madrid (CCC-UAM) for allocation of computer time. Work supported by the MICINN Projects No. FIS2010-15127, No. FIS2010-18847, No. CTQ2010-17006, No. FIS-2010-19609C09-00, No. ACI2008-0777, No. 2010C-07-25200, and No. CSD2007-00010, the CAM program NANOBIOMAGNET S2009/MAT1726 and the Gobierno Vasco-UPV/EHU Project No. IT-366-07. S.B. acknowledges financial support from MEC under FPU Grant No. AP-2007-00157. D.S. acknowledges financial support from the FPI-UAM grant program. *fernando.martin@uam.es

${ }^{1}$ K. Novoselov, A. Geim, S. Morozov, D. Jiang, M. Katsnelson, I. Grigorieva, S. Dubonos, and A. Firsov, Nature (London) 438, 197 (2005).

${ }^{2}$ A. H. Castro Neto, F. Guinea, N. M. R. Peres, K. S. Novoselov, and A. K. Geim, Rev. Mod. Phys. 81, 109 (2009).

${ }^{3}$ J. Wintterlin and M. L. Bocquet, Surf. Sci. 603, 1841 (2009).

${ }^{4}$ G. Giovannetti, P. A. Khomyakov, G. Brocks, V. M. Karpan, J. van den Brink, and P. J. Kelly, Phys. Rev. Lett. 101, 026803 (2008).
${ }^{5}$ S. Marchini, S. Günther, and J. Wintterlin, Phys. Rev. B 76, 075429 (2007).

${ }^{6}$ P. W. Sutter, J.-I. Flenge, and E. A. Sutter, Nat. Mater. 7, 406 (2008).

${ }^{7}$ Y. Pan, H. Zhang, D. Shi, J. Sun, S. Du, F. Liu, and H.-J. Gao, Adv. Mater. 20, 1 (2008).

${ }^{8}$ A. L. Vásquez de Parga, F. Calleja, B. Borca, M. C. G. Passeggi Jr., J. J. Hinarejos, F. Guinea, and R. Miranda, Phys. Rev. Lett. 100, 056807 (2008). 
${ }^{9}$ E. Miniussi, M. Pozzo, A. Baraldi, E. Vesselli, R. R. Zhan, G. Comelli, T. O. Menteş, M. A. Niño, A. Locatelli, S. Lizzit et al., Phys. Rev. Lett. 106, 216101 (2011).

${ }^{10}$ B. Wang, M. Caffio, C. Bromley, H. Früchtl, and R. Schaub, ACS Nano 10, 5773 (2010).

${ }^{11}$ E. N. Voloshina, Y. S. Dedkov, S. Torbrügge, A. Thissen, and M. Fonin, Appl. Phys. Lett. 100, 241606 (2012).

${ }^{12}$ I. Pletikosić, M. Kralj, P. Pervan, R. Brako, J. Coraux, A. T. N'Diaye, C. Busse, and T. Michely, Phys. Rev. Lett. 102, 056808 (2009).

${ }^{13}$ K. Hermann, J. Phys.: Condens. Matter 24, 314210 (2012).

${ }^{14}$ A. B. Preobrajenski, M. L. Ng, A. S. Vinogradov, and N. Mårtensson, Phys. Rev. B 78, 073401 (2008).

${ }^{15}$ M. C. Payne, M. P. Teter, D. C. Allan, T. A. Arias, and J. D. Joannopoulos, Rev. Mod. Phys. 64, 1045 (1992).

${ }^{16}$ B. Wang, M. L. Bocquet, S. Marchini, S. Günther, and J. Wintterlin, Phys. Chem. Chem. Phys. 10, 3530 (2008).

${ }^{17}$ B. Wang, S. Günther, J. Wintterlin, and M. L. Bocquet, New J. Phys. 12, 043041 (2010).

${ }^{18}$ B. Borca, S. Barja, M. Garnica, D. Sanchez-Portal, V. Silkin, E. V. Chulkov, C. F. Hermanns, J. J. Hinarejos, A. L. V. de Parga, A. Arnau et al., Phys. Rev. Lett. 105, 036804 (2010).

${ }^{19}$ D. Stradi, S. Barja, C. Díaz, M. Garnica, B. Borca, J. J. Hinarejos, D. Sánchez-Portal, M. Alcamí, A. Arnau, A. L. V. de Parga et al., Phys. Rev. Lett. 106, 186102 (2011).

${ }^{20}$ D. Stradi, S. Barja, C. Díaz, M. Garnica, B. Borca, J. J. Hinarejos, D. Sánchez-Portal, M. Alcamí, A. Arnau, A. L. V. de Parga et al., Phys. Rev. B 85, 121404(R) (2012).

${ }^{21}$ C. Busse, P. Lazić, R. Djemour, J. Coraux, T. Gerber, N. Atodiresei, V. Caciuc, R. B. A. T. N'Diaye, S. Blügel, J. Zegenhagen et al., Phys. Rev. Lett. 107, 036101 (2011).

${ }^{22}$ M. Iannuzzi and J. Hutter, Surf. Sci. 605, 1360 (2011).

${ }^{23}$ A. T. N'Diaye, S. Bleikamp, P. J. Feibelman, and T. Michely, Phys. Rev. Lett. 97, 215501 (2006).

${ }^{24}$ T. Brugger, S. Günther, B. Wang, H. Dil, M.-L. Bocquet, J. Osterwalder, J. Wintterlin, and T. Greber, Phys. Rev. B 79, 045407 (2009).

${ }^{25}$ S. J. Altenburg, J. Kröger, T. O. Wehling, B. Sachs, A. I. Lichtenstein, and R. Berndt, Phys. Rev. Lett. 108, 206805 (2012).

${ }^{26}$ S.-M. Choi, S.-H. Jhi, and Y.-W. Son, Phys. Rev. B 81, 081407 (2010).

${ }^{27}$ A. Varykhalov, J. Sánchez-Barriga, A. M. Shikin, C. Biswas, E. Vescovo, A. Rybkin, D. Marchenko, and O. Rader, Phys. Rev. Lett. 101, 157601 (2008).

${ }^{28}$ A. Grüneis and K. K. D. V. Vyalikh, New J. Phys. 11, 073050 (2011).

${ }^{29}$ S. M. Kozlov, F. Viñes, and A. Görling, J. Phys. Chem. C 116, 7360 (2012).

${ }^{30}$ W. Zhao, S. M. Kozlov, O. Höfert, K. Gotterbarm, M. P. A. Lorenz, F. V. nes, C. Papp, A. Görling, and H.-P. Steinrück, J. Phys. Chem. Lett. 2, 759 (2011).

${ }^{31}$ H. G. Zhang and T. Greber, Phys. Rev. Lett. 105, 219701 (2010).

${ }^{32}$ B. Borca, S. Barja, M. Garnica, D. Sánchez-Portal, V. M. Silkin, E. V. Chulkov, C. F. Hermanns, J. J. Hinarejos, A. L. Vázquez de Parga, A. Arnau et al., Phys. Rev. Lett. 105, 219702 (2010).

${ }^{33}$ D. Martoccia, P. R. Willmott, T. Brugger, M. Björck, S. Günther, C. M. Schlepütz, A. Cervellino, S. A. Pauli, B. D. Patterson, S. Marchini et al., Phys. Rev. Lett. 101, 126102 (2008).
${ }^{34}$ W. Moritz, B. Wang, M.-L. Bocquet, T. Brugger, T. Greber, J. Wintterlin, and S. Günther, Phys. Rev. Lett. 104, 136102 (2010).

${ }^{35}$ D. Martoccia, M. Björck, C. M. Schlepütz, T. Brugger, S. A. Pauli, B. D. Patterson, T. Greber, and P. R. Willmott, New J. Phys. 12, 043028 (2010).

${ }^{36}$ B. Borca, S. Barja, M. Garnica, M. Minniti, A. Politano, J. M. Rodriguez-García, J. J. Hinarejos, D. Farías, A. L. V. de Parga, and R. Miranda, New J. Phys. 12, 093018 (2010).

${ }^{37}$ M. Gyamfi, T. Eelbo, M. Waśniowska, and R. Wiesendanger, Phys. Rev. B 83, 153418 (2011).

${ }^{38}$ H. G. Zhang, H. Hu, Y. Pan, J. H. Mao, M. Gao, H. M. Guo, S. X. Du, T. Greber, and H.-J. Gao, J. Phys.: Condens. Matter 22, 302001 (2010).

${ }^{39}$ G. Kresse and J. Hafner, Phys. Rev. B 47, 558 (1993).

${ }^{40}$ G. Kresse and D. Joubert, Phys. Rev. B 59, 1758 (1999).

${ }^{41}$ J. P. Perdew, K. Burke, and M. Ernzerhof, Phys. Rev. Lett. 77, 3865 (1996).

${ }^{42}$ S. Grimme, J. Comp. Chem. 27, 1787 (2006).

${ }^{43}$ P. E. Blöchl, Phys. Rev. B 50, 17953 (1994).

${ }^{44}$ H. J. Monkhorst and J. D. Pack, Phys. Rev. B 13, 5188 (1976).

${ }^{45}$ M. Methfessel and A. T. Paxton, Phys. Rev. B 40, 3616 (1989).

${ }^{46}$ J. Tersoff and D. R. Hammann, Phys. Rev. Lett. 50, 1998 (1983).

${ }^{47}$ C. Corriol, J. Hager, R. Matzdorf, and A. Arnau, Surf. Sci. 600, 4310 (2006).

${ }^{48}$ P. Sutter, M. S. Hybertsen, J. T. Sadowski, and E. Sutter, Nano Lett. 9, 2654 (2009).

${ }^{49}$ S. Grimme, J. Antony, S. Ehrlich, and H. Krieg, J. Chem. Phys. 132, 154104 (2010).

${ }^{50}$ B. Cordero, V. Gómez, A. E. Platero-Prats, M. Revés, J. Echeverría, E. Cremades, F. Barragán, and S. Alvarez, Dalton Trans. 21, 2832 (2008).

${ }^{51}$ M. Hasegawa and K. Nishidate, Phys. Rev. B 70, 205431 (2004).

${ }^{52}$ X. Wu, M. C. Vargas, S. Nayak, V. Lotrich, and G. Scoles, J. Chem. Phys. 115, 8748 (2001).

${ }^{53}$ D. C. Langreth, B. I. Lundqvist, S. D. Chakarova-Käck, V. R. Cooper, M. Dion, P. Hyldgaard, A. Kelkkanen, J. Kleis, L. Kong, S. Li et al., J. Phys.: Condens. Matter 21, 084203 (2009).

${ }^{54}$ F. Ortmann, F. Bechstedt, and W. G. Schmidt, Phys. Rev. B 73, 205101 (2006)

${ }^{55}$ T. Olsen, J. Yan, J. J. Mortensen, and K. S. Thygesen, Phys. Rev. Lett. 107, 156401 (2011).

${ }^{56}$ F. Mittendorfer, A. Garhofer, J. Redinger, J. Klimeš, J. Harl, and G. Kresse, Phys. Rev. B 84, 201401(R) (2011).

${ }^{57}$ G. Mercurio, E. R. McNellis, I. Martin, S. Hagen, F. Leyssner, S. Soubatch, J. Meyer, M. Wolf, P. Tegeder, F. S. Tautz et al., Phys. Rev. Lett. 104, 036102 (2010).

${ }^{58}$ V. G. Ruiz, W. Liu, E. Zojer, M. Scheffler, and A. Tkatchenko, Phys. Rev. Lett. 108, 146103 (2012).

${ }^{59}$ K. Tonigold and A. Groß, J. Chem. Phys. 132, 224701 (2010).

${ }^{60}$ R. Zacharia, H. Ulbricht, and T. Hertel, Phys. Rev. B 69, 155406 (2004).

${ }^{61}$ Y. X. Zhao and I. L. Spain, Phys. Rev. B 40, 993 (1989).

${ }^{62}$ D. Jiang, M.-H. Du, and S. Dai, J. Chem. Phys. 130, 074705 (2009).

${ }^{63}$ B. Wang and M. L. Bocquet, Nanoscale 4, 4687 (2012). 
${ }^{64}$ A. L. Vásquez de Parga, F. Calleja, B. Borca, M. C. G. Passegi, Jr., J. J. Hinarejos, F. Guinea, and R. Miranda, Phys. Rev. Lett. 101, 099704 (2008).

${ }^{65}$ W. A. Hofer, A. S. Foster, and A. L. Shluger, Rev. Mod. Phys. 75, 1287 (2003).

${ }^{66}$ V. M. Silkin, J. Zhao, F. Guinea, E. V. Chulkov, P. M. Echenique, and H. Petek, Phys. Rev. B 80, 121408 (2009).
${ }^{67}$ N. Armbrust, J. Güdde, P. Jakob, and U. Höfer, Phys. Rev. Lett. 108, 056801 (2012).

${ }^{68}$ R. D. Muiño, D. Sánchez-Portal, V. M. Silkin, E. V. Chulkov, and P. M. Echenique, Proc. Natl. Acad. Sci. USA 108, 971 (2011).

${ }^{69}$ S. Lizzit, R. Larciprete, P. Lacovig, K. L. Kostov, and D. Menzel, ACS Nano 7, 4359 (2013). 